JOURNAL OF THE

AMERICAN MATHEMATICAL SOCIETY

Volume 23, Number 2, April 2010, Pages 345-382

S 0894-0347(09)00655-9

Article electronically published on December 24, 2009

\title{
ON THE BREAKDOWN CRITERION IN GENERAL RELATIVITY
}

\author{
SERGIU KLAINERMAN AND IGOR RODNIANSKI
}

\section{INTRODUCTION}

This paper is concerned with the problem of a geometric criterion for the breakdown of solutions $(\mathbf{M}, \mathbf{g})$ of the vacuum Einstein equations

$$
\mathbf{R}_{\alpha \beta}(\mathbf{g})=0 .
$$

To describe the problem we assume that a part of space-time $\mathcal{M}_{*} \subset \mathbf{M}$ is foliated by the level hypersurface of a time function $t$, monotonically increasing towards the future, with lapse $n$ and second fundamental form $k$ defined by

$$
k(X, Y)=-\mathbf{g}\left(\mathbf{D}_{X} \mathbf{T}, Y\right), \quad n=(-\mathbf{g}(\mathbf{D} t, \mathbf{D} t))^{-1 / 2},
$$

where $\mathbf{T}$ is the future unit normal to $\Sigma_{t}, \mathbf{D}$ is the space-time covariant derivative associated with $\mathbf{g}$, and $X, Y$ are tangent to $\Sigma_{t}$. Let $\Sigma_{0}$ be a fixed leaf of the $t$ foliation, corresponding to $t=t_{0}$. We shall refer to $\Sigma_{0}$ as the initial slice. We assume that the space-time region $\mathcal{M}_{*}$ is globally hyperbolic, i.e. every causal curve from a point $p \in \mathcal{M}_{*}$ intersects $\Sigma_{0}$ at precisely one point. We also assume that the initial slice satisfies the following assumption.

A 1. There exists a finite covering of $\Sigma_{0}$ by a finite number of charts $U$ such that for any fixed chart, the induced metric $g$ satisfies

$$
\Delta_{0}^{-1}|\xi|^{2} \leq g_{i j}(x) \xi_{i} \xi_{j} \leq \Delta_{0}|\xi|^{2}, \quad \forall x \in U
$$

with $\Delta_{0}$ a fixed positive number.

We consider the following two situations:

(1) The surfaces $\Sigma_{t}$ are asymptotically flat and maximal,

$$
\operatorname{tr} k=0 .
$$

(2) The surfaces $\Sigma_{t}$ are compact, of Yamabe type -1, and of constant, negative mean curvature. They form what is called a (CMC) foliation

$$
\operatorname{tr} k=t, \quad t<0 \text {. }
$$

Though our methods apply equally well to both situations we shall only consider here the latter case, which is somewhat easier to treat due to the compactness of the level surfaces $\Sigma_{t}$. We shall thus assume in what follows that the region $\mathcal{M}_{*}$ is

Received by the editors February 4, 2008.

2010 Mathematics Subject Classification. Primary 35J10.

The first author is partially supported by NSF grant DMS-0070696.

The second author is partially supported by NSF grant DMS-0702270.

(C)2009 American Mathematical Society 
equal to $\bigcup_{t \in\left[t_{0}, t_{*}\right)} \Sigma_{t}$, with $t_{*}<0$. We can also assume that the initial hypersurface $\Sigma_{0}$ corresponds to $t_{0}=-1$.

Remark. In the second case the CMC conjecture asserts that it should be possible to extend the foliation, in a smooth manner, to all values of $\operatorname{tr} k=t<0$; see And and references therein.

Given $p \in \mathcal{M}_{*}$ we can define a pointwise norm $|\Pi(p)|$ of any space-time tensor $\Pi$ via the decomposition

$$
X=-X^{0} \mathbf{T}+\underline{X}, \quad X \in T \mathcal{M}_{*}, \quad \underline{X} \in T \Sigma_{t} .
$$

We denote by $\|\Pi(t)\|_{L^{p}}$ the $L^{p}$-norm of $\Pi$ on $\Sigma_{t}$. More precisely,

$$
\|\Pi(t)\|_{L^{p}}=\int_{\Sigma_{t}}|\Pi|^{p} d v_{g}
$$

with $d v_{g}$ the volume element of the metric $g$ of $\Sigma_{t}$. The main result of this paper is the following theorem.

Theorem 1.1 (Main theorem). Let (M,g) be a globally hyperbolic development of $\Sigma_{0}$ foliated by the CMC level hypersurfaces of a time function $t<0$, such that $\Sigma_{0}$ corresponds to the level surface $t=t_{0}$. Assume that $\Sigma_{0}$ satisfies $\mathbf{A 1}$. Then the first time $T_{*}<0$, with respect to the t-foliation, of a breakdown is characterized by the condition

$$
\limsup _{t \rightarrow T_{*}^{-}}\left(\|k(t)\|_{L^{\infty}}+\|\nabla \log n(t)\|_{L^{\infty}}\right)=\infty .
$$

More precisely the space-time together with the foliation $\Sigma_{t}$ can be extended beyond any value $t_{*}<0$ for which

$$
\sup _{t \in\left[t_{0}, t_{*}\right)}\|k(t)\|_{L^{\infty}}+\|\nabla \log n(t)\|_{L^{\infty}}=\Delta_{0}<\infty .
$$

Remark 1.2. In the case of a maximal foliation the left-hand side of (5) should also contain the quantity $\left\|n^{-1}\right\|_{L^{\infty}}$ as in that case the lapse $n$ does not have an a priori uniform lower bound.

Condition (5) can be reformulated in terms of the deformation tensor of the future unit normal $\mathbf{T}, \pi={ }^{(\mathbf{T})} \pi=\mathcal{L}_{\mathbf{T}} \mathbf{g}$. By a simple calculation, expressed relative to an orthonormal frame $e_{0}=\mathbf{T}, e_{1}, e_{2}, e_{3}$, we find

$$
\pi_{00}=0, \quad \pi_{0 i}=n^{-1} \nabla_{i} n, \quad \pi_{i j}=-2 k_{i j} .
$$

Consistent with the statement of the main theorem we assume that $\mathbf{T}$ is an approximate Killing vector field in the following sense:

A2. There exists a constant $\Delta_{0}$ such that

$$
\sup _{t \in\left[t_{0}, t_{*}\right)}\|\pi(t)\|_{L^{\infty}} \leq \Delta_{0} .
$$

In addition to the constant $\Delta_{0}$ in $\mathbf{A} \mathbf{1}, \mathbf{A} 2$ we introduce another constant $\mathcal{R}_{0}$ which plays an important role in the proof, which bounds the $L^{2}$-norm of the spacetime curvature tensor $\mathbf{R}$ on $\Sigma_{0}$,

$$
\left\|\mathbf{R}\left(t_{0}\right)\right\|_{L^{2}\left(\Sigma_{0}\right)} \leq \mathcal{R}_{0} .
$$

To prove our main theorem we have to show that if assumptions A1 and A2 are satisfied, then the space-time $\mathcal{M}_{*}$ can be extended beyond $t_{*}$. We want to 
emphasize that Theorem 1.1 is a large data result; indeed, we make no smallness assumptions on the constants $\Delta_{0}$ and $\mathcal{R}_{0}$.

Our theorem is connected and partially motivated by the following three earlier breakdown criteria results:

1. The first is a result of M. Anderson, And, who showed that a breakdown can be tied to the condition that

$$
\limsup _{t \rightarrow t_{*}^{-}}\|\mathbf{R}(t)\|_{L^{\infty}}=\infty .
$$

Our result can be viewed as complementary. It is clear however that the condition (41) is formally weaker as it refers only to the second fundamental form $k$ and the lapse $n$ which requires one degree less of differentiability. Moreover a condition on the boundedness of the $L^{\infty}$-norm of $\mathbf{R}$ exhausts all the dynamical degrees of freedom of the equations. Indeed, once we know that $\|\mathbf{R}(t)\|_{L^{\infty}}$ is finite, one can find bounds for $n, \nabla n$ and $k$ on $\Sigma_{t}$ purely by elliptic estimates. This is certainly not true in our case.

2. Our result can also be compared to the well-known Beale-Kato-Majda, BKM], criterion for the breakdown of solutions of the incompressible Euler equation

$$
\partial_{t} v+(v \cdot \nabla) v=-\nabla p, \quad \operatorname{div} v=0,
$$

with smooth initial data at $t=t_{0}$. A routine application of the energy estimates shows that the solution $v$ blows up if and only if

$$
\int_{t_{0}}^{t_{*}}\|\nabla v(t)\|_{L^{\infty}} d t=\infty
$$

The Beale-Kato-Majda result improves the blow-up criterion by replacing it with the following condition on the vorticity $\omega=\operatorname{curl} v$ :

$$
\int_{t_{0}}^{t_{*}}\|\omega(t)\|_{L^{\infty}} d t=\infty
$$

To relate $\nabla v$ and $\omega$ one observes that

$$
\operatorname{div} v=0, \quad \operatorname{curl} v=\omega
$$

forms an elliptic system for $v$ in terms of $\omega$. Thus $\nabla v$ can be expressed in terms of $\omega$ via a singular integral operator, i.e. a zero-order pseudodifferential operator:

$$
\nabla v=P^{0}(\omega) \text {. }
$$

Although $P^{0}$ does not define a bounded map $L^{\infty} \rightarrow L^{\infty}$ it can be shown that (11) is sufficient to reduce the breakdown condition (9) to the more satisfying one (10), in terms of the vorticity alone.

Similarly, in the case of the Einstein equations energy estimates, expressed relative to a special system of coordinates (such as wave coordinates), we show that breakdown does not occur unless

$$
\int_{t_{0}}^{t_{*}}\|\partial \mathbf{g}(t)\|_{L^{\infty}} d t=\infty
$$

This condition however is not geometric as it depends on the choice of a full coordinate system. Observe that both the spatial derivatives of the lapse $\nabla n$ and the components of the second fundamental form, $k_{i j}=-\frac{1}{2} n^{-1} \partial_{t} g_{i j}$, can be viewed as components of $\partial \mathrm{g}$. 
Note however that after prescribing $k$ and $\nabla n$ we are still left with many more degrees of freedom in determining $\partial \mathbf{g}$. The fundamental difficulty that one needs to overcome is that of deriving bounds for $\mathbf{R}$ using only bounds for $\|\nabla \log n(t)\|_{L^{\infty}}+$ $\|k(t)\|_{L^{\infty}}$ and geometric information on the initial hypersurface $\Sigma_{0}$. Clearly this cannot be done by elliptic estimates alone. Thus, as opposed to both the results of M. Anderson and Beale-Kato-Majda, it is far less obvious that a condition such as (41) can cover all dynamic degrees of freedom of the Einstein equations. Despite the formal similarity with the previous results mentioned above, the proof of Theorem 1.1 requires a conceptually different treatment.

3. Finally, the result whose proof is closest in spirit to ours and which has played the main motivating role in developing our approach is the proof of global regularity of solutions of the Yang-Mills equations in $\mathbb{R}^{3+1}$ by Eardley and Moncrief; see [EM1, EM2]. To explain the connection of their result to ours we review below its main ideas.

Recall that the curvature tensor $\mathbf{F}_{\alpha \beta}$ of a Yang-Mills connection $\lambda_{\alpha} d x^{\alpha}$, with values in the Lie algebra $s u(N)$ is a critical point of the Yang-Mills functional

$$
Y M[\mathbf{F}]=\int_{\mathbb{R}^{3+1}} \operatorname{Tr}\left({ }^{\star} \mathbf{F} \wedge \mathbf{F}\right)
$$

and satisfies the wave equation,

$$
\square_{(\lambda)} \mathbf{F}=\mathbf{F} \star \mathbf{F},
$$

where $\square_{(\lambda)}$ denotes the covariant wave operator,

$$
\square_{(\lambda)} \mathbf{F}=\mathbf{D}^{\alpha} \mathbf{D}_{\alpha} \mathbf{F}=\square \mathbf{F}+[\lambda, \partial \mathbf{F}]+[\partial \lambda, \mathbf{F}]+[\lambda,[\lambda, \mathbf{F}]],
$$

denotes the usual d'Alembertian in $\mathbb{R}^{3+1}$ and $\mathbf{D}_{a}=\partial_{\alpha}+\left[\lambda_{\alpha}, \cdot\right]$, the gauge covariant derivative. Since the Minkowski space-time metric

$$
\mathbf{m}=-d t^{2}+\delta_{i j} d x^{i} d x^{j}
$$

is static (in particular $n=1$ and $k=0$ ) the energy of $\mathbf{F}$ associated with the energy-momentum tensor $\mathbf{Q}[\mathbf{F}]_{\alpha \beta}=\mathbf{F}_{\alpha}{ }_{\alpha} \mathbf{F}_{\beta \lambda}+{ }^{\star} \mathbf{F}_{\alpha}{ }^{\lambda \star} \mathbf{F}_{\beta \lambda}$ and vector field $\mathbf{T}=\partial_{t}$ is conserved. In particular, the flux of energy $\mathcal{F}_{p}$ through the null boundary $\mathcal{N}^{-}(p)$ of the domain of dependence $\mathcal{J}^{-}(p)$ of an arbitrary point $p$ can be bounded by the energy of the initial data which we denote by $I_{0}$. We assume that smooth data for $F$ is prescribed at $t=0$ and restrict $\mathcal{J}^{-}(p)$ and $\mathcal{N}^{-}(p)$ to $t \geq 0$. We recall that the flux has the form, $\mathcal{F}_{p}=\left(\int_{\mathcal{N}^{-}(p)} \mathbf{Q}[\mathbf{F}](L, \mathbf{T})\right)^{\frac{1}{2}}$ with $L=-\partial_{t}+\partial_{r}$ the null geodesic generator of $\mathcal{N}^{-}(p)$ normalized by the condition $\langle L, \mathbf{T}\rangle=1$.

The proof of the global regularity of solutions of the Yang-Mills equations is based on the boundedness of the flux $\mathcal{F}_{p} \leq I_{0}<\infty$. Here is a summary of the main steps.

1. Rewrite (12) in the form $\square \mathbf{F}=\mathbf{F} \star \mathbf{F}-\left(\square_{(\lambda)}-\square\right) \mathbf{F}$. Using the explicit representation, in $\mathbb{R}^{3+1}$, of solutions to the inhomogeneous wave equation, we deduce, for all points $p$, with $t>0$,

$$
\begin{gathered}
\mathbf{F}(p)=(4 \pi)^{-1} \int_{\mathcal{N}^{-}(p ; \delta)} r^{-1} \mathbf{F} \star \mathbf{F}+\mathbf{F}^{(0)}(p ; \delta) \\
-(4 \pi)^{-1} \int_{\mathcal{N}^{-}(p ; \delta)} r^{-1}\left(\square_{(\lambda)}-\square\right) \mathbf{F} .
\end{gathered}
$$

Here $\mathcal{N}^{-}(p, \delta)$ represents the portion of the null cone $\mathcal{N}^{-}(p)$ included in the time slab $[t(p)-\delta, t(p))$, with $t(p)$ the value of the time parameter at $p$. Also $r$ is 
the distance, in the Euclidean sense, to the vertex $p$ and $\mathbf{F}^{(0)}(p ; \delta)$ represents a homogeneous solution to the wave equation whose initial data at $t=t(p)-\delta$ coincide with those of $\mathbf{F}$.

2. Ignore, for a moment, the presence of the third term on the right-hand side of (13). Using the explicit form of the nonlinear term $\mathbf{F} \star \mathbf{F}$ one notices that at least one component of the product can be estimated by the flux $\mathcal{F}_{p}$ of $\mathbf{F}$ through the null hypersurface $\mathcal{N}^{-}(p)$. Denoting $|\mathbf{F}|=\sum_{\alpha \beta}\left|\mathbf{F}_{\alpha \beta}\right|$, we have by a simple estimate 1

$$
\begin{aligned}
\mid\left(\mathbf{F}(p)-\mathbf{F}^{(0)}(p ; \delta) \mid\right. & \lesssim \mathcal{F}_{p}\left(\int_{\mathcal{N}^{-}(p ; \delta)} r^{-2}\right)^{1 / 2}\|\mathbf{F}\|_{L^{\infty}\left(\mathcal{J}^{-}(p ; \delta)\right)} \\
& \lesssim \delta^{1 / 2} \mathcal{F}_{p}\|\mathbf{F}\|_{L^{\infty}\left(\mathcal{J}^{-}(p, \delta)\right)},
\end{aligned}
$$

where $\|\mathbf{F}\|_{L^{\infty}\left(\mathcal{J}^{-}(p, \delta)\right)}$ denotes the sup-norm of $|\mathbf{F}|$ for all points in the domain of dependence $\mathcal{J}^{-}(p)$ of $p$ intersected with the slab $[t(p)-\delta, t(p)]$. Therefore, we deduce that if $\delta^{1 / 2} \cdot \mathcal{F}_{p}$ is sufficiently small, then for any $t \geq 0$,

$$
\|\mathbf{F}(t)\|_{L^{\infty}} \lesssim\|\mathbf{F}(t-\delta)\|_{L^{\infty}}+\|\mathbf{D F}(t-\delta)\|_{L^{\infty}} .
$$

3. Arguing recursively and using the standard local existence theorem for the Yang-Mills system 2 one can find bounds for all components of the curvature tensol 3 $\mathbf{F}(p)$ depending only on the fact that $\mathcal{F}_{p}$ is uniformly bounded and the initial data data $\mathbf{F}(0)$ is smooth.

4. One can show that (14) remains true even as we take into consideration the presence of the third term in (13). Consider for example what could be, potentially, the most dangerous term,

$$
\int_{\mathcal{N}^{-}(p ; \delta)} r^{-1} \lambda \cdot \partial \mathbf{F}
$$

Here we have to hope that we can integrate by parts to transfer the derivative from $\mathbf{F}$ to $\lambda$. This can only be done if $\lambda \cdot \partial$ is tangential to the light cone $\mathcal{N}^{-}(p)$. Miraculously, this can be achieved by taking $\lambda$ in the Cronström gauge; that is, one assumes that the connection 1-form $\lambda$ satisfies

$$
(x-y)^{\alpha} \lambda_{\alpha}=0,
$$

where $x^{\alpha}$ are the space-time coordinates of $p$ and $y^{\alpha}$ those of a point $q \in \mathcal{N}^{-}(p)$. With this choice, after integration by parts, one can treat all the remaining terms in $\left(\square_{(\lambda)}-\square\right) \mathbf{F}$ roughly in the same way as the main term $F \star F$. To show this one has to observe that the value of $\lambda$ at any point $q$ in the domain of dependence of $p$ can be estimated by $\|\mathbf{F}\|_{L^{\infty}\left(\mathcal{J}^{-}(p, \delta)\right)}$. This leads to the same estimate (14) as stated in the lemma above.

5. In Kl-Ma the global regularity result was reproved by strengthening the classical local existence result to $\lambda \in H^{1}\left(\mathbb{R}^{3}\right)$ and $E \in L^{2}\left(\mathbb{R}^{3}\right)$, which is at the same regularity level as the energy norm. That required, instead of the pointwise estimates (14), a new generation of $L^{4}$-type estimates, called bilinear. The premise of the [Kl-Ma] approach was the fact that, once we have a local existence result which depends only on the energy norm of the initial data, global existence can be easily derived by a simple continuation argument.

\footnotetext{
${ }^{1}$ Here and throughout the paper the notation $F \lesssim G$ means $F \leq c G$ with $c$ a universal constant.

${ }^{2}$ In a given gauge such as the Coulomb or the Lorenz gauge.

${ }^{3}$ Once bounds are established for $F(p)$ one can proceed in the same manner to derive bounds for derivatives of $\mathbf{F}$ at $p$.
} 
6. In Kl-Ro5 we have developed a gauge-independent approach to the proof of the Eardley-Moncrief result. The approach is based on a Kirchoff-Sobolev parametrix for $\square_{(\lambda)}$, similar to the one we use in this paper, which replaces (13) by a gauge-invariant formula depending, implicitly 4 only on the values of $\mathbf{F}$ along $\mathcal{N}^{-}(p)$.

This paper was motivated in part by the desire to adapt the Eardley-Moncrief argument5 to General Relativity. The above discussion indicates that the EardleyMoncrief proof relies on two independent ingredients: conservation of energy and pointwise bounds on curvature, which depend only on the flux and initial data. Since the analogue of the Yang-Mills energy in General Relativity (the Bel-Robinson energy) is not conserved, one can only hope to reproduce the second part of the Eardley-Moncrief argument and prove a conditional regularity result which states, roughly, that smooth solutions of the Einstein equations, in a vacuum, remain smooth, and can therefore be continued, as long as an integral quantity, we call the flux of curvature, remains bounded. The possibility of such a result first became apparent to us in a discussion with V. Moncrief 6 Such a result could also be deduced, in principle, from the stronger bounded $L^{2}$-curvature conjecture, according to which the initial value problem is well posed for initial data sets with $L^{2}$-bounds on its curvature. In this paper we actually take a step closer to implementing the full analogue of the Eardley-Moncrief result. Rather than imposing a direct condition on the finiteness of the Bel-Robinson energy and curvature flux we formulate conditions (perhaps more natural albeit more restrictive) which control the extent to which the energy is not conserved. These conditions, which form our breakdown criterion, involve uniform bounds on the second fundamental form $k$ and derivatives of the lapse $n$.

In what follows we give a short summary of how the main ideas in the proof of the Eardley-Moncrief result for Yang-Mills can be adapted to GR.

1. The curvature tensor $\mathbf{R}$ of a $(3+1)$-dimensional vacuum spacetime $(\mathbf{M}, \mathbf{g})$, see (11), satisfies a wave equation of the form

$$
\square_{\mathbf{g}} \mathbf{R}=\mathbf{R} \star \mathbf{R}
$$

where $\square_{\mathbf{g}}$ denotes the covariant wave operator $\square_{\mathbf{g}}=\mathbf{D}^{\alpha} \mathbf{D}_{\alpha}$.

2. The Bel-Robinson tensor has the form

$$
\mathbf{Q}[\mathbf{R}]_{\alpha \beta \gamma \delta}=\mathbf{R}_{\alpha \lambda \gamma \mu} \mathbf{R}_{\beta \delta \delta}^{\lambda \mu}+{ }^{\star} \mathbf{R}_{\alpha \lambda \gamma \mu}{ }^{\star} \mathbf{R}_{\beta \delta}^{\lambda \mu}
$$

and satisfies $\mathbf{D}^{\delta} \mathbf{Q}_{\alpha \beta \gamma \delta}=0$. It can thus be used to derive energy and flux estimates for the curvature tensor R. As opposed to the case of the Yang-Mills theory, however, in General Relativity the background metric is a dynamic variable itself and thus does not admit, in general, Killing fields (and in particular a time-like Killing field). This means that we cannot associate conserved quantities to a divergence free Bel-Robinson tensor. It is at this point where we need crucially our approximate Killing condition A2. Indeed that condition suffices to derive bounds for both energy and flux associated to the curvature tensor R. Using the Bel-Robinson

\footnotetext{
${ }^{4}$ Through transport equations along the null boundary of the causal past of $p$.

${ }^{5}$ Adapting [Kl-Ma] to General Relativity is the goal of the bounded $L^{2}$-curvature conjecture; see $\mathrm{Kl}$.

${ }^{6} \mathrm{~V}$. Moncrief has been independently pursuing the analogy between the Einstein and YangMills equations by developing an integral representation of the curvature tensor in General Relativity based on the Hadamard-Friedlander method (as in [Fried]); see [M].
} 
energy momentum tensor $\mathbf{Q}$ the energy associated to a slice $\Sigma_{t}$ is defined by the integral

$$
\mathcal{E}(t)=\left(\int_{\Sigma_{t_{2}}} \mathbf{Q}[\mathbf{R}](\mathbf{T}, \mathbf{T}, \mathbf{T}, \mathbf{T})\right)^{\frac{1}{2}}
$$

while the flux, through the null boundary $\mathcal{N}^{-}(p)$ of the domain of dependence (or causal past) $\mathcal{J}^{-}(p)$ of a point $p$, is given by the integral

$$
\mathcal{F}^{-}(p)=\left(\int_{\mathcal{N}^{-}(p)} \mathbf{Q}[\mathbf{R}](L, \mathbf{T}, \mathbf{T}, \mathbf{T})\right)^{\frac{1}{2}},
$$

where $L$ is the null geodesic generator of $\mathcal{N}^{-}(p)$ normalized at the vertex $p$ by $\langle L, \mathbf{T}\rangle=1$.

As in the case of the Yang-Mills equations it is precisely the boundedness of the flux of curvature that plays a crucial role in our analysis. In General Relativity the flux has an even more fundamental role as it is also needed to control the geometry of the very object it is defined on, i.e. the boundary of the causal past of $p$. This boundary, unlike in the case of Minkowski space, is not determined a priori but depends in fact on the space-time we are trying to control.

3. In the construction of a parametrix for (16) we cannot, in any meaningful way, approximate $\square_{\mathbf{g}}$ by the flat d'Alembertian $\square$. To deduce a formula analogous to (14) one might try to proceed by the geometrics optics construction of parametrices for $\square_{\mathbf{g}}$, as developed in Fried]. Such an approach would require additional bounds on the background geometry, determined by the metric $\mathbf{g}$, incompatible with the limited assumption A2 and the implied finiteness of the curvature flux. We rely instead on a geometric version, which we develop in Kl-Ro5, of the Kirchoff-Sobolev formula, in the spirit of that used by Sobolev in Sob] and $\mathrm{Y}$. Choquet-Bruhat in $\mathrm{Br} 7$ Applying that formula to equation (16) we obtain the following analogue of the formula (14):

$$
\mathbf{R}(p)=-\int_{\mathcal{N}^{-}(p ; \delta)} \mathbf{A} \cdot(\mathbf{R} \star \mathbf{R})+\mathcal{E}+\int_{\mathcal{N}^{-}(p ; \delta)} \operatorname{Err} \cdot \mathbf{R},
$$

where $\mathbf{A}$ is a 4-covariant, 4-contravariant tensor defined as a solution of a transport equation along $\mathcal{N}^{-}(p, \delta)$ with appropriate (blowing-up) initial data at the vertex $p, \mathcal{N}^{-}(p ; \delta)$ denotes the portion of the null boundary $\mathcal{N}^{-}(p)$ in the time interval $[t(p)-\delta, t(p)]$ and the error term Err depends only on the extrinsic geometry of $\mathcal{N}^{-}(p ; \delta)$. The term $\mathcal{E}$ depends, in principle, only on the properties of the space-time in the interval $[t(p)-\delta, t(p)-\delta / 2]$.

4. As in the Yang-Mills setting the structure of the term $\mathbf{R} \star \mathbf{R}$ allows us to estimate one of the curvature terms by the flux of curvature:

$$
\begin{aligned}
\left|\int_{\mathcal{N}^{-}(p, \delta)} \mathbf{A} \cdot(\mathbf{R} \star \mathbf{R})\right| & \lesssim \mathcal{F}^{-}(p) \cdot\|\mathbf{R}\|_{L^{\infty}\left(\mathcal{N}^{-}(p, \delta)\right)} \cdot\|\mathbf{A}\|_{L^{2}\left(\mathcal{N}^{-}(p, \delta)\right)} \\
& \lesssim \delta^{1 / 2} \cdot \mathcal{F}^{-}(p) \cdot\|\mathbf{R}\|_{L^{\infty}\left(\mathcal{N}^{-}(p, \delta)\right)},
\end{aligned}
$$

\footnotetext{
${ }^{7}$ It is extremely important that the error term generated by our parametrix depends only on the geometry of the boundary of the causal past of a point. This feature is absent in all previous constructions. A similar feature is also present in the recent work of Moncrief; see [M].
} 
provided that

$$
\|\mathbf{A}\|_{L^{2}\left(\mathcal{N}^{-}(p ; \delta)\right)} \lesssim \delta^{1 / 2} .
$$

Neglecting, for a moment, the third integral in (19) we can thus expect to prove a result analogous to that in (14); see Proposition 5.11.

Theorem 1.3. There exists a sufficiently small $\delta>0$ and a large constant $C$, depending only on $\Delta_{0}$ in assumptions $\mathbf{A} \mathbf{1}$ and $\mathbf{A 2}$ as well as $\mathcal{R}_{0}$ in (8) such that for all $t_{0} \leq t<t_{*}$,

$$
\|\mathbf{R}(t)\|_{L^{\infty}} \lesssim \delta^{-1} C \sup _{t-2 \delta \leq t^{\prime} \leq t-\delta / 2}\left(\left\|\mathbf{R}\left(t^{\prime}\right)\right\|_{L^{2}}+\|\mathbf{D R}(t)\|_{L^{2}}+\left\|\mathbf{D}^{2} \mathbf{R}(t)\right\|_{L^{2}}\right) .
$$

5. The proof of (20) depends on verifying (21). In addition, to estimate the third term in (19), we need to provide estimates for tangential derivatives of $\mathbf{A}$ and other geometric quantities associated to the null hypersurfaces $\mathcal{N}^{-}(p)$. In particular, this requires showing that $\mathcal{N}^{-}(p)$ remains a smooth (not merely Lipschitz) hypersurface in the time slab $(t(p)-\delta, t(p)]$ for some $\delta>0$ dependent only on the constants $\Delta_{0}$ and $\mathcal{R}_{0}$. Thus to prove the desired theorem we have to show that all geometric quantities, arising in the parametrix construction, can be estimated only in terms of the flux of the curvature $\mathcal{F}_{p}^{-}$along $\mathcal{N}^{-}(p)$ and our main assumption A1. Yet, to start with, it is not even clear that we can provide a lower bound for the radius of injectivity of $\mathcal{N}^{-}(p)$. In other words the congruence of null geodesics, initiating at $p$, may not be controllabl 8 only in terms of the curvature flux. Typically, in fact, lower bounds for the radius of conjugacy of a null hypersurface in a Lorentzian manifold are only available in terms of the sup-norm of the curvature tensor $\mathbf{R}$ along the hypersurface, while the problem of short, intersecting, null geodesics appears not to be fully understood even in that context. The situation is similar to that in Riemannian geometry, exemplified by Cheeger's theorem, where pointwise bounds on sectional curvature are sufficient to control the radius of conjugacy, but to prevent the occurrence of short geodesic loops one needs to assume in addition an upper bound on the diameter and a lower bound on the volume of the manifold.

In a sequence of papers, [Kl-Ro1] $\mathrm{Kl}-\mathrm{Ro} 3$, see also Wang 9 , we have proved lower bounds on the geodesic radius of conjugacy of null hypersurfaces. The methods developed in those papers can be adapted to also prove lower bounds on the radius of conjugacy with respect to the time parameter 10 . It may however be possible that the radius of conjugacy of the null congruence is bounded from below and yet there are past null geodesics from a point $p$ intersecting again at points arbitrarily close, with respect to the time parameter $t$, to $p$. In [Kl-Ro4] we have shown that this cannot happen in a space-time satisfying our conditions $\mathbf{A} \mathbf{1}$ and A2. Thus the combined results of Kl-Ro1-Kl-Ro4 allow us to derive a lower bound on the radius of injectivity of $\mathcal{N}^{-}(p)$ depending only on $\Delta_{0}$.

\footnotetext{
${ }^{8}$ Different null geodesics of the congruence may intersect, or the congruence itself may have conjugate points, arbitrarily close to $p$.

${ }^{9}$ In Kl-Ro1- Kl-Ro3 we have considered the case of the congruence of outgoing future null geodesics initiating on a 2 -surface $S_{0}$ embedded in a space-like hypersurface $\Sigma_{0}$. The extension of our results to null cones from a point forms the subject of Qian Wang's Princeton $2006 \mathrm{PhD}$ thesis, see Wang.

${ }^{10}$ The results in [Kl-Ro1- Kl-Ro3] and Wang were proved with respect to the geodesic foliation. In this paper, as well as in [Kl-Ro4, we rely on an extension of these results to the foliation on $\mathcal{N}^{-}(p)$ induced by the space-like foliation $\Sigma_{t}$.
} 
6. As in the case of Yang-Mills equations, one can use the result of Theorem 1.3. together with the classical local existence result for the Einstein equations, such as that in $[\mathrm{C}-\mathrm{K}$, to show that solutions can be extended as long as the bounds on ${ }^{(\mathbf{T})} \pi$ hold true.

Finally we would like to point out possible refinements of our Main Theorem 1.1 We expect that one should be able to replace the pointwise condition A2 with the integral condition

$$
\int_{t_{0}}^{t_{*}}\|\pi(t)\|_{L^{\infty}}^{2} d t<\infty
$$

Moreover it may be possible to improve the result even further by eliminating the term $\nabla \log n$ in (44) or (23) and requiring instead only a pointwise bound on $n$.

\section{Constant mean CURVATURe Foliations}

As described in the introduction, $\left(\mathcal{M}_{*}, \mathbf{g}\right)$ denotes a Lorentzian manifold of the form $\mathcal{M}_{*}=I \times \Sigma$, where $\Sigma$ is a three-dimensional, compact, connected, orientable smooth manifold foliated by a CMC foliation $\Sigma_{t}$ with lapse $n$ and second fundamental $k$,

$$
n=(-\mathbf{g}(\mathbf{D} t, \mathbf{D} t))^{-1 / 2}, \quad k(X, Y)=\mathbf{g}\left(\mathbf{D}_{X} \mathbf{T}, Y\right),
$$

where $\mathbf{T}$ denotes the future unit normal to $\Sigma_{t}$. The time interval $I=\left[t_{0}, t_{*}\right)$, where $t_{0}=-1$ and $t_{*}<0$.

We decompose a space-time vector field $X$ relative to the unit timelike $\mathbf{T}$,

$$
X=X^{0} \mathbf{T}+\underline{X}, \quad\langle\mathbf{T}, \underline{X}\rangle=0 .
$$

We define the positive definite Riemannian metric,

$$
h(X, Y)=X^{0} \cdot Y^{0}+g(\underline{X}, \underline{Y}),
$$

where $g$ denotes the metric induced on $\Sigma_{t}$. We can also write (25) in the form

$$
h_{\alpha \beta}=\mathbf{g}_{\alpha \beta}+2 \mathbf{T}_{\alpha} \mathbf{T}_{\beta} .
$$

Given a space-time tensor $U$ we denote by $|U|$ its norm with respect to the metric $h$. More precisely, if $U$ is an $m$-covariant tensor, then

$$
|U|^{2}=h^{i_{1} j_{1}} \ldots h^{i_{m} j_{m}} U_{i_{1} \ldots i_{m}} U_{j_{1} \ldots j_{m}} .
$$

The following bound follows immediately from our main assumption (5),

$$
|\mathbf{D T}| \lesssim \Delta_{0}
$$

Since $\mathbf{D}_{\gamma} h_{\alpha \beta}=2\left(\mathbf{D}_{\gamma} \mathbf{T}_{\alpha} \mathbf{T}_{\beta}+\mathbf{T}_{\alpha} \mathbf{D}_{\gamma} \mathbf{T}_{\beta}\right)$ we have $|\mathbf{D} h| \leq 4|\mathbf{D T}|$. Therefore,

$$
|\mathbf{D} h| \lesssim \Delta_{0} .
$$

Also, since the components of the deformation tensor $\pi={ }^{(\mathbf{T})} \pi=\mathcal{L}_{\mathbf{T}} \mathbf{g}$ are given by

$$
\pi_{00}=0, \quad \pi_{0 i}=n^{-1} \nabla_{i} n, \quad \pi_{i j}=n^{-1} \partial_{t} g_{i j}=-2 k_{i j}
$$

we have

$$
{ }^{(\mathbf{T})} \pi \mid \lesssim \Delta_{0}
$$


Given two tensors $U, V$ we shall denote by $U \cdot V$ any tensor which is obtained from the tensor product of $U$ and $V$ by taking contractions with respect to the space-time metric g. Clearly,

$$
|U \cdot V| \lesssim|U| \cdot|V|
$$

For any coordinate chart $\mathcal{O}$, with coordinates $x=\left(x^{1}, x^{2}, x^{3}\right)$, we denote by $\left(x^{0}=t, x^{1}, x^{2}, x^{3}\right)$ the transported coordinates on $I \times \mathcal{O}$ obtained by following the integral curves of $\mathbf{T}$. In these coordinates the metric $\mathbf{g}$ takes the form

$$
\mathbf{g}=-n^{2} d t^{2}+g_{i j} d x^{i} d x^{j}
$$

Relative to these coordinates $t, x$, we have the equations

$$
\begin{aligned}
\partial_{t} g_{i j} & =-2 n k_{i j}, \\
\partial_{t} k_{i j} & =-\nabla_{i} \nabla_{j} n+n\left(R_{i j}+\operatorname{tr}_{g} k k_{i j}-2 k_{i a} k^{a}{ }_{j}\right)
\end{aligned}
$$

with $R_{i j}$ the Ricci curvature of the induced metric $g$ on $\Sigma_{t}$. We also have the constraint equations,

$$
\begin{gathered}
R-|k|^{2}+(\operatorname{tr} k)^{2}=0, \\
\nabla^{j} k_{i j}=\nabla_{i} \operatorname{tr} k .
\end{gathered}
$$

In view of the constant mean curvature condition on the foliation $\Sigma_{t}$ we can always reparametrize $t$ so that

$$
\operatorname{tr}_{g} k=t
$$

As mentioned in the introduction, we can assume that the initial hypersurface $\Sigma_{0}$ corresponds to the value $t=t_{0}=-1$. In view of (33), (34) and (36) we deduce the lapse equation,

$$
\Delta n=|k|^{2} n-1
$$

At a point $p$ of minimum for $n$ we must have $|k|^{2} n-1 \geq 0$. Therefore, at $p$, $n \geq|k(p)|^{-2}$. On the other hand, since $|k|^{2}=|\hat{k}|^{2}+\frac{1}{3}(\operatorname{tr} k)^{2}$, at a point of maximum we have $|\hat{k}|^{2} n+\frac{1}{3}(\operatorname{tr} k)^{2} n-1 \leq 0$. Therefore,

$$
\frac{1}{\|k(t)\|_{L^{\infty}}^{2}} \leq n \leq \frac{3}{t^{2}} .
$$

Observe also that, since $\partial_{t} \log (\operatorname{det} g)=-2 n \operatorname{tr} k=-2 n t$,

$$
\frac{d}{d t}\left|\Sigma_{t}\right|=\frac{d}{d t} \int_{\Sigma_{0}} \sqrt{\operatorname{det} g} d x=-\int_{\Sigma_{0}} n t \sqrt{\operatorname{det} g} d x,
$$

where $\left|\Sigma_{t}\right|$ denotes the volume of the compact manifold $\Sigma_{t}$. Thus,

$$
0 \leq \frac{d}{d t}\left|\Sigma_{t}\right| \leq 3\left|\Sigma_{t}\right||t|^{-1}
$$

As a consequence of (36) the ratio of the volumes of $\left|\Sigma_{t}\right|$ and $\left|\Sigma_{0}\right|$ can be estimated by

$$
1 \leq \frac{\left|\Sigma_{t}\right|}{\left|\Sigma_{0}\right|} \leq \frac{\left|t_{0}\right|^{3}}{|t|^{3}}
$$


Therefore, since $t_{0}=-1$, we have proved

Proposition 2.1. For all $-1=t_{0} \leq t<t_{*}<0$ we have the bounds

$$
\frac{1}{\|k(t)\|_{L^{\infty}}^{2}} \leq n \leq \frac{3}{t^{2}} .
$$

Moreover, if $\left|\Sigma_{t}\right|$ denotes the volume of $\Sigma_{t}$ and $\Sigma_{0}=\Sigma_{t_{0}}$,

$$
\left|\Sigma_{0}\right| \leq\left|\Sigma_{t}\right| \leq \frac{1}{|t|^{3}}\left|\Sigma_{0}\right| \lesssim|t|^{-3} \text {. }
$$

2.2. Coordinate estimates. We recall the following lemma; see Lemma 2.2 in Kl-Ro4.

Lemma 2.3. If $\Sigma_{0}$ is compact and satisfies $\mathbf{A} 1$ of the introduction, there must exist a number $\rho_{0}>0$ such that every point $y \in \Sigma_{0}$ admits a neighborhood $B$, included in a neighborhood chart $U$, such that $B$ is precisely the Euclidean ball $B=B_{\rho_{0}}^{(e)}(y)$ relative to the local coordinates in $U$.

Proof. 1 See the proof of Lemma 2.2 in [Kl-Ro4.

Next we recall the result of Proposition 4.1 in Kl-Ro4.

Proposition 2.4. If assumptions $\mathbf{A} 1$ and $\mathbf{A} 2$ are satisfied, then there exists a large constant $C=C\left(\Delta_{0}\right)$ such that in the region $\mathcal{M}_{*}$,

$$
C^{-1}|\xi|^{2} \leq g_{i j}(t, x) \xi^{i} \xi^{j} \leq C|\xi|^{2}, \quad \forall x \in U .
$$

Proof. For convenience we reproduce the proof given in Kl-Ro4. We fix a coordinate chart $U$ and consider the transported coordinates $t, x^{1}, x^{2}, x^{3}$ on $I \times U$. Thus $\partial_{t} g_{i j}=-2 n k_{i j}$. Let $X$ be a time-independent vector on $\mathbf{M}$ tangent to $\Sigma_{t}$. Then,

$$
\partial_{t} g(X, X)=-\frac{1}{2} n k(X, X)
$$

Clearly,

$$
|n k(X, X)| \leq|n k|_{g}|X|_{g}^{2} \leq\|n k(t)\|_{L^{\infty}}|X|_{g}^{2}
$$

with $|k|_{g}^{2}=g^{a c} g^{b d} k_{a b} k_{c d}$ and $|X|_{g}^{2}=X^{i} X^{j} g_{i j}=g(X, X)$. Therefore, since $\partial_{t}|X|_{g}^{2}=$ $\partial_{t} g(X, X)$,

$$
-2\|n k(t)\|_{L^{\infty}}|X|_{g}^{2} \leq \partial_{t}|X|_{g}^{2} \leq 2\|n k(t)\|_{L^{\infty}}|X|_{g}^{2}
$$

Thus,

$$
|X|_{g_{0}} e^{-2 \int_{t_{0}}^{t}\|n k(\tau)\|_{L} \infty d \tau} \leq|X|_{g_{t}}^{2} \leq|X|_{g_{0}} e^{2 \int_{t_{0}}^{t}\|n k(\tau)\|_{L} \infty d \tau},
$$

from which (41) immediately follows.

2.5. Sobolev inequalities. The properties of local transported coordinates established in the previous section can be used to prove the following Sobolev inequality for scalar functions.

Proposition 2.6. Assume assumptions A1 and A2 are satisfied. There exists a constant $C$ depending only on $\Delta_{0}$ such that for every smooth scalar function on $\Sigma_{t}$, $t_{0} \leq t<t^{*}$,

$$
\|f\|_{L^{\frac{3}{2}}\left(\Sigma_{t}\right)} \leq C\left(\|\nabla f\|_{L^{1}\left(\Sigma_{t}\right)}+\|f\|_{L^{1}\left(\Sigma_{t}\right)}\right) .
$$


Proof. By a partition of unity we may assume that $f$ has compact support in a local chart $V=\Sigma_{t} \cap(I \times U)$ of transported coordinates $t, x=\left(x^{1}, x^{2}, x^{3}\right)$. Then, writing

$$
\begin{gathered}
f(x)=\int_{-\infty}^{x^{1}} \partial_{1} f\left(y, x^{2}, x^{3}\right) d y=\int_{-\infty}^{x^{2}} \partial_{2} f\left(x^{1}, y, x^{3}\right) d y=\int_{-\infty}^{x^{3}} \partial_{3} f\left(x^{1}, x^{2}, y\right) d y, \\
|f(x)|^{3 / 2} \leq\left(\int_{-\infty}^{x^{1}}\left|\partial_{1} f\left(y, x^{2}, x^{3}\right)\right| d y\right. \\
\left.\cdot \int_{-\infty}^{x^{2}}\left|\partial_{2} f\left(x^{1}, y, x^{3}\right)\right| d y \int_{-\infty}^{x^{3}}\left|\partial_{1} f\left(x^{1}, x^{2}, y\right)\right| d y\right)^{1 / 2} .
\end{gathered}
$$

Thus, by Hölder,

$$
\int_{V}|f(x)|^{3 / 2} d x \leq\left(\int_{V}|\nabla f(x)| d x\right)^{3 / 2} .
$$

Therefore, since in view of (41) we have $C^{-3 / 2} \leq \sqrt{|g|} \leq C^{3 / 2}$,

$$
\left(\int_{V}|f(x)|^{3 / 2} \sqrt{|g|} d x\right)^{\frac{1}{2}} \lesssim \int_{V}|\nabla f(x)| \sqrt{|g|} d x,
$$

which proves (42) as desired.

Corollary 2.7. For any smooth tensor field $F$ on $\Sigma_{t}$ and any $2 \leq p \leq 6$,

$$
\|F\|_{L^{p}\left(\Sigma_{t}\right)} \leq C\left(\|\nabla F\|_{L^{2}\left(\Sigma_{t}\right)}^{3 / 2-3 / p}\|F\|_{L^{2}\left(\Sigma_{t}\right)}^{3 / p-1 / 2}+\|F\|_{L^{2}\left(\Sigma_{t}\right)}\right) .
$$

Proof. We have

$$
\begin{aligned}
\|F\|_{L^{p}}^{2 p / 3} & =\left\||F|^{2 p / 3}\right\|_{L^{3 / 2}} \leq C\left(\left\|\nabla|F|^{2 p / 3}\right\|_{L^{1}}+\left\||F|^{2 p / 3}\right\|_{L^{1}}\right) \\
& \leq C\left(\|\nabla F\|_{L^{2}}+\|F\|_{L^{2}}\right) \cdot\left\||F|^{2 p / 3-1}\right\|_{L^{2}} \\
& \leq C\left(\|\nabla F\|_{L^{2}}+\|F\|_{L^{2}}\right) \cdot\left(\|F\|_{L^{\frac{4 p-6}{3}}}\right)^{\frac{4 p-6}{6}}
\end{aligned}
$$

In the particular case when $p=6$, we derive

$$
\|F\|_{L^{6}}^{4} \leq C\left(\|\nabla F\|_{L^{2}}+\|F\|_{L^{2}}\right) \cdot\|F\|_{L^{6}}^{3} .
$$

Therefore,

$$
\|F\|_{L^{6}} \leq C\left(\|\nabla F\|_{L^{2}}+\|F\|_{L^{2}}\right) .
$$

Similarly, for $p=3$,

$$
\|F\|_{L^{3}}^{2} \leq C\left(\|\nabla F\|_{L^{2}}+\|F\|_{L^{2}}\right) \cdot\|F\|_{L^{2}}
$$

and thus,

$$
\|F\|_{L^{3}} \leq C\left(\|\nabla F\|_{L^{2}}+\|F\|_{L^{2}}\right)^{1 / 2} \cdot\|F\|_{L^{2}}^{1 / 2} .
$$

The general case follows by interpolation.

Here is another useful simple calculus inequality which we will make use of.

Lemma 2.8. Let $F$ be a tensor field on a compact Riemannian manifold. Then,

$$
\|\nabla F\|_{L^{4}} \leq 2\left\|\nabla^{2} F\right\|_{L^{2}}^{1 / 2}\|F\|_{L^{\infty}}^{1 / 2} .
$$


Proof. After an integration by parts and Hölder,

$$
\int_{\Sigma}|\nabla F|^{4} \leq 4\left\|\nabla^{2} F\right\|_{L^{2}}\|\nabla F\|_{L^{4}}^{2}\|F\|_{L^{\infty}}
$$

Hence,

$$
\|\nabla F\|_{L^{4}}^{2} \leq 4\left\|\nabla^{2} F\right\|_{L^{2}}\|F\|_{L^{\infty}} .
$$

Remark. We cannot use transported coordinates to derive a Sobolev inequality of the form

$$
\|f\|_{L^{\infty}} \lesssim\left\|\nabla^{2} f\right\|_{L^{2}}+\|f\|_{L^{2}}
$$

even in the case of a scalar function $f$. Indeed, the standard Sobolev inequality in a coordinate chart $U$ provides

$$
\|f\|_{L^{\infty}(U)} \lesssim \sum_{i, j=1}^{3}\left\|\partial_{i} \partial_{j} f\right\|_{L^{2}(U)}+\|f\|_{L^{2}(U)} .
$$

On the other hand, $\nabla_{i} \nabla_{j} f=\partial_{i} \partial_{j} f-\Gamma_{i j}^{l} \partial_{l} f$ and therefore we cannot derive the desired estimate without a bound for the $L^{3}$-norm of $\Gamma$. Unfortunately, the only way to estimate $\Gamma$ is by differentiating the equation $\partial_{t} g=-2 n k$ from which we could only bound its $L^{2}$-norm. To get around this difficulty we need a better system of coordinates. In [Kl-Ro4] we have proved a slightly more general version of the following:

Theorem 2.9. Assume that $\mathcal{M}_{*}$ is globally hyperbolic and satisfies the assumptions A1 and A2 as well as (8). Then, for any $\epsilon>0$, there exists $r_{0}>0$, depending only on $\epsilon, \Delta_{0}, \mathcal{R}_{0}, t_{*}$, such that on any geodesic ball $B_{r} \subset \Sigma_{t}, r \leq r_{0}$, centered at a point $p_{t} \in \Sigma_{t}$, there exist local coordinates relative to which the metric $g_{t}$ satisfies the conditions

$$
\begin{aligned}
(1+\epsilon)^{-1} \delta_{i j} \leq g_{i j} & \leq(1+\epsilon) \delta_{i j} \\
r \int_{B_{r}(p)}\left|\partial^{2} g_{i j}\right|^{2} d v_{g} & \leq \epsilon .
\end{aligned}
$$

As a corollary we derive the following version of the Sobolev inequality.

Corollary 2.10. Given a smooth scalar function $f$ on $\Sigma_{t}$ we have

$$
\left.\|f\|_{L^{\infty}\left(\Sigma_{t}\right)} \leq C\left\|\nabla^{2} f\right\|_{L^{2}\left(\Sigma_{t}\right)}+\|f\|_{L^{2}\left(\Sigma_{t}\right)}\right)
$$

with $C>$ a universal constant, i.e. depending only on the fundamental constants $\Delta_{0}, \mathcal{R}_{0}, t_{*}$.

\section{BAsic CuRvature EnERgy estimates}

3.1. General procedure. We recall the general procedure to derive energy estimates for $\mathbf{R}$; see section 7.1 in $[\mathrm{C}-\mathrm{K}$. First let $W$ denote a Weyl field, i.e., a four covariant tensor traceless tensor $W_{\alpha \beta \gamma \delta}$ satisfying all the algebraic symmetries of the curvature tensor $\mathbf{R}$. Let

$$
\mathbf{Q}[W]_{\alpha \beta \gamma \delta}=W_{\alpha \lambda \gamma \mu} W_{\beta \delta}^{\lambda \mu}+{ }^{\star} W_{\alpha \lambda \gamma \mu}{ }^{\star} W_{\beta \delta}^{\lambda \mu} .
$$


Given a vector field $X$ we denote $P_{\alpha}=\mathbf{Q}[W]_{\alpha \beta \gamma \delta} X^{\beta} X^{\gamma} X^{\delta}$. By a straightforward calculation,

$$
\mathbf{D}^{\alpha} P_{\alpha}=\mathbf{D i v} \mathbf{Q}[W] X^{\beta} X^{\gamma} X^{\delta}+\frac{3}{2} \mathbf{Q}_{\alpha \beta \gamma \delta}{ }^{(X)} \pi^{\alpha \beta} X^{\gamma} X^{\delta},
$$

where ${ }^{(X)} \pi$ is the deformation tensor of $X$. Therefore, integrating on the slab $\bigcup_{t^{\prime} \in\left[t_{0}, t\right]} \Sigma_{t^{\prime}}$ we derive the following.

Proposition 3.2. Let $\mathbf{Q}=\mathbf{Q}[W]$ be the Bel-Robinson tensor of a Weyl field $W$. Then,

$$
\begin{aligned}
\int_{\Sigma_{t}} \mathbf{Q}(X, X, X, \mathbf{T})= & \int_{\Sigma_{0}} \mathbf{Q}(X, X, X, \mathbf{T})+\int_{t_{0}}^{t} \int_{\Sigma_{t^{\prime}}} \operatorname{Div} \mathbf{Q}(X, X, X) n d v_{g} \\
& +\frac{3}{2} \int_{t_{0}}^{t} \int_{\Sigma_{t^{\prime}}} \mathbf{Q}_{\alpha \beta \gamma \delta}{ }^{(X)} \pi^{\alpha \beta} X^{\gamma} X^{\delta} n d v_{g}
\end{aligned}
$$

with $d v_{g}$ denoting the volume element on $\Sigma_{t}$.

The following proposition is an immediate consequence; see also section 5 in Kl-Ro4 for a proof. One simply needs to apply the proposition above for $X=\mathbf{T}$ together with the positivity of $\mathbf{Q}(\mathbf{T}, \mathbf{T}, \mathbf{T}, \mathbf{T})$ and the uniform bounds for ${ }^{(\mathbf{T})} \pi$ and $n$.

Proposition 3.3. Under assumption A2, there exists a constant $C=C\left(\Delta_{0}, t_{*}\right)$ such that, for any $t_{0} \leq t<t_{*}<0$,

$$
\|\mathbf{R}(t)\|_{L^{2}} \leq C \mathcal{R}_{0},
$$

where $\mathcal{R}_{0}$ is the constant defined by (8).

Definition 3.4. In what follows we extend the usual notation $A \lesssim B$ to include inequalities $A \leq c B$, where $c=c\left(t_{*}, \Delta_{0}, \mathcal{R}_{0}\right)$ is a constant which depends on our fundamental constants $t_{*}, \Delta_{0}$ and $\mathcal{R}_{0}$; see also the footnote following (14).

In particular, in view of Proposition 3.3 we can write

$$
\|\mathbf{R}(t)\|_{L^{2}} \lesssim \mathcal{R}_{0} \lesssim 1
$$

3.5. Wave equation for the curvature tensor. Recall the Bianchi identities,

$$
\mathbf{D}_{[\sigma} \mathbf{R}_{\alpha \beta] \gamma \delta}=0
$$

or, equivalently since $\mathbf{R}_{\alpha \beta}=0$,

$$
\mathbf{D}^{\delta} \mathbf{R}_{\alpha \beta \gamma \delta}=0 .
$$

Differentiating (50) once more and taking the trace, we derive

$$
\square \mathbf{R}_{\alpha \beta \gamma \delta}+\mathbf{D}^{\sigma} \mathbf{D}_{\alpha} \mathbf{R}_{\beta \sigma \gamma \delta}+\mathbf{D}^{\sigma} \mathbf{D}_{\beta} \mathbf{R}_{\sigma \alpha \gamma \delta}=0 .
$$

Now, in view of (51), commuting covariant derivatives,

$$
\begin{aligned}
\mathbf{D}^{\sigma} \mathbf{D}_{\alpha} \mathbf{R}_{\beta \sigma \gamma \delta} & =\mathbf{R}_{\beta}{ }^{\mu \sigma}{ }_{\alpha} \mathbf{R}_{\mu \sigma \gamma \delta}+\mathbf{R}_{\gamma}{ }^{\mu \sigma}{ }_{\alpha} \mathbf{R}_{\beta \sigma \mu \delta}+\mathbf{R}_{\delta}{ }^{\mu \sigma}{ }_{\alpha} \mathbf{R}_{\beta \sigma \gamma \mu}, \\
\mathbf{D}^{\sigma} \mathbf{D}_{\beta} \mathbf{R}_{\alpha \sigma \gamma \delta} & =\mathbf{R}_{\alpha}{ }_{\alpha}{ }_{\beta} \mathbf{R}_{\mu \sigma \gamma \delta}+\mathbf{R}_{\gamma}{ }^{\mu \sigma}{ }_{\beta} \mathbf{R}_{\alpha \sigma \mu \delta}+\mathbf{R}_{\delta}{ }^{\mu \sigma}{ }_{\beta} \mathbf{R}_{\alpha \sigma \gamma \mu} .
\end{aligned}
$$

Hence,

$$
\begin{aligned}
\mathbf{D}^{\sigma} \mathbf{D}_{\alpha} \mathbf{R}_{\beta \sigma \gamma \delta}+\mathbf{D}^{\sigma} \mathbf{D}_{\beta} \mathbf{R}_{\sigma \alpha \gamma \delta}= & \mathbf{R}_{\mu \sigma \gamma \delta}\left(\mathbf{R}_{\beta}^{\mu \sigma}{ }_{\alpha}-\mathbf{R}_{\alpha \beta}^{\mu \sigma}\right) \\
& -\mathbf{R}_{\alpha \sigma \gamma \mu}\left(\mathbf{R}_{\delta}^{\mu \sigma}{ }_{\beta}+\mathbf{R}_{\beta}{ }_{\beta}{ }_{j}\right) \\
& -\mathbf{R}_{\alpha \sigma \mu \delta}\left(\mathbf{R}_{\beta}^{\sigma \mu}{ }_{\gamma}+\mathbf{R}_{\gamma}{ }_{\beta}{ }_{\beta}\right)
\end{aligned}
$$


Thus introducing the notation,

$$
\begin{aligned}
(\mathbf{R} \star \mathbf{R})_{\alpha \beta \gamma \delta}= & -\mathbf{R}_{\mu \sigma \gamma \delta}\left(\mathbf{R}_{\beta}^{\mu \sigma}{ }_{\alpha}-\mathbf{R}_{\alpha}^{\mu \sigma}{ }_{\beta}\right)+\mathbf{R}_{\alpha \sigma \gamma \mu}\left(\mathbf{R}_{\delta}{ }_{\beta}{ }_{\beta}+\mathbf{R}_{\beta}{ }^{\sigma \mu} \delta\right. \\
& +\mathbf{R}_{\alpha \sigma \mu \delta}\left(\mathbf{R}_{\beta}{ }^{\sigma \mu}{ }_{\gamma}+\mathbf{R}_{\gamma}{ }^{\mu \sigma}{ }_{\beta}\right),
\end{aligned}
$$

we derive

$$
\square \mathbf{R}=\mathbf{R} \star \mathbf{R} .
$$

Clearly $W=\mathbf{R} \star \mathbf{R}$ is a Weyl field; i.e., it satisfies all the algebraic symmetries of the curvature tensor plus the traceless condition $W_{\alpha \mu \beta}^{\mu}=0$.

3.6. Energy estimates for higher derivatives. To estimate the first derivatives of $\mathbf{R}$ we shall use the covariant wave equation (53). Recall the positive definite space-time metric $h$ defined by (25). Given a tensor field $U_{\alpha_{1} \ldots \alpha_{m}}$ we write, for simplicity,

$$
\begin{aligned}
h^{I J} U_{I} U_{J} & =h^{\alpha_{1} \beta_{1}} \ldots h^{\alpha_{m} \beta_{m}} U_{\alpha_{1} \ldots \alpha_{m}} U_{\beta_{1} \ldots \beta_{m}}, \\
U_{I} & =U_{\alpha_{1} \ldots \alpha_{m}}, \quad U_{J}=U_{\beta_{1} \ldots \beta_{m}}, \quad h^{I J}=h^{\alpha_{1} \beta_{1}} \ldots h^{\alpha_{m} \beta_{m}} .
\end{aligned}
$$

Consider the energy-momentum type tensor $\mathbf{Q}_{\alpha \beta}^{(w)}$ associated with the covariant wave operator $\square$ acting on tensors,

$$
\mathbf{Q}^{(w)}[U]_{\alpha \beta}:=h^{I J} \mathbf{D}_{\alpha} U_{I} \mathbf{D}_{\beta} U_{J}-\frac{1}{2} \mathbf{g}_{\alpha \beta} h^{I J} \mathbf{g}^{\mu \nu} \mathbf{D}_{\mu} U_{I} \mathbf{D}_{\nu} U_{J}
$$

We have

$$
\begin{aligned}
\mathbf{D}^{\beta} \mathbf{Q}^{(w)}[U]_{\alpha \beta}= & h^{I J} \mathbf{D}_{\alpha} U_{I} \mathbf{D}^{\beta} \mathbf{D}_{\beta} U_{J}+h^{I J} \mathbf{D}^{\beta} \mathbf{D}_{\alpha} U_{I} \mathbf{D}_{\beta} U_{J} \\
& -\mathbf{g}_{\alpha \beta} h^{I J} \mathbf{g}^{\mu \nu} \mathbf{D}^{\beta} \mathbf{D}_{\mu} U_{I} \mathbf{D}_{\nu} U_{J} \\
& +D^{\beta} h^{I J} \mathbf{D}_{\alpha} U_{I} \mathbf{D}_{\beta} U_{J}-\frac{1}{2} \mathbf{g}_{\alpha \beta} D^{\beta} h^{I J} \mathbf{g}^{\mu \nu} \mathbf{D}_{\mu} U_{I} \mathbf{D}_{\nu} U_{J} \\
= & h^{I J} \mathbf{D}_{\alpha} U_{I}\left(\square U_{J}\right)+h^{I J}\left(\mathbf{D}_{\beta} \mathbf{D}_{\alpha} U_{I}-\mathbf{D}_{\alpha} \mathbf{D}_{\beta} U_{I}\right) \mathbf{D}^{\beta} U_{J} \\
& +D^{\beta} h^{I J}\left(\mathbf{D}_{\alpha} U_{I} \mathbf{D}_{\beta} U_{J}-\frac{1}{2} \mathbf{g}_{\alpha \beta} \mathbf{g}^{\mu \nu} \mathbf{D}_{\mu} U_{I} \mathbf{D}_{\nu} U_{J}\right) .
\end{aligned}
$$

Consequently, in view of (29),

$$
\left|\mathbf{D Q}^{(w)}[U]\right| \lesssim|\mathbf{D} U||\square U|+|\mathbf{R}||U||\mathbf{D} U|+\Delta_{0}|\mathbf{D} U|^{2} .
$$

Therefore, since

$$
\mathbf{D}^{\beta}\left(\mathbf{Q}^{(w)}[U]_{\alpha \beta} \mathbf{T}^{\alpha}\right)=D^{\beta} \mathbf{T}^{\alpha} \mathbf{Q}^{(w)}[U]_{\alpha \beta}+T^{\beta} \mathbf{D}^{\beta} \mathbf{Q}^{(w)}[U]_{\alpha \beta},
$$

we derive

$$
\left|\mathbf{D}^{\beta}\left(\mathbf{Q}^{(w)}[U]_{\alpha \beta} \mathbf{T}^{\alpha}\right)\right| \lesssim|\mathbf{D} U||\square U|+|\mathbf{R}||U||\mathbf{D} U|+\Delta_{0}|\mathbf{D} U|^{2} .
$$

On the other hand,

$$
\begin{aligned}
\mathbf{Q}^{(w)}[U](\mathbf{T}, \mathbf{T}) & =\frac{1}{2} h^{I J}\left(\mathbf{D}_{0} U_{I} \mathbf{D}_{0} U_{J}+\sum_{l=1}^{k} \mathbf{D}_{l} U_{I} \mathbf{D}_{l} U_{J}\right) \\
& =\frac{1}{2}|\mathbf{D} U|^{2} .
\end{aligned}
$$


Integrating (55) we derive

$$
\begin{aligned}
\int_{\Sigma_{t}}|\mathbf{D} U|^{2} & =2 \int_{\Sigma_{t}} \mathbf{Q}^{(w)}[U](\mathbf{T}, \mathbf{T}) \\
& \leq \int_{\Sigma_{t_{0}}}|\mathbf{D} U|^{2}+\int_{t_{0}}^{t} \int_{\Sigma_{t^{\prime}}}\left|\mathbf{D}^{\beta}\left(\mathbf{Q}^{(w)}[U]_{\alpha \beta} \mathbf{T}^{\alpha}\right)\right| \\
& \lesssim \int_{t_{0}}^{t} \int_{\Sigma_{t^{\prime}}}\left(|\mathbf{D} U||\square U|+|\mathbf{R}||U||\mathbf{D} U|+\Delta_{0}|\mathbf{D} U|^{2}\right) .
\end{aligned}
$$

Applying this to $U=\mathbf{R}$ and using the equation (53) we obtain

$$
\begin{aligned}
\|\mathbf{D R}(t)\|_{L^{2}}^{2} \lesssim & \left\|\mathbf{D R}\left(t_{0}\right)\right\|_{L^{2}}^{2}+\Delta_{0} \int_{t_{0}}^{t}\left\|\mathbf{D R}\left(t^{\prime}\right)\right\|_{L^{2}}^{2} d t^{\prime} \\
& +\int_{t_{0}}^{t}\left\|\mathbf{D R}\left(t^{\prime}\right)\right\|_{L^{2}}\left\|\mathbf{R}\left(t^{\prime}\right)\right\|_{L^{2}}\left\|\mathbf{R}\left(t^{\prime}\right)\right\|_{L^{\infty}} d t^{\prime} \\
\lesssim & \left\|\mathbf{D R}\left(t_{0}\right)\right\|_{L^{2}}^{2}+\Delta_{0} \int_{t_{0}}^{t}\left\|\mathbf{D R}\left(t^{\prime}\right)\right\|_{L^{2}}^{2} d t^{\prime} \\
& +\mathcal{R}_{0} \int_{t_{0}}^{t}\left\|\mathbf{D R}\left(t^{\prime}\right)\right\|_{L^{2}}\left\|\mathbf{R}\left(t^{\prime}\right)\right\|_{L^{\infty}} d t^{\prime}
\end{aligned}
$$

Therefore in order to get an a priori estimate for $\|\mathbf{D R}(t)\|_{L^{2}}$ it suffices to prove an estimate for the $L^{\infty}$-norm of $\mathbf{R}$. More precisely,

Proposition 3.7. Assume that the assumptions A1, A2 hold true. Then the following derivative curvature estimates hold true for all $t_{0} \leq t<t_{*}$,

$$
\|\mathbf{D R}(t)\|_{L^{2}}^{2} \leq C\left(\left\|\mathbf{D R}\left(t_{0}\right)\right\|_{L^{2}}^{2}+\int_{t_{0}}^{t}\left\|\mathbf{R}\left(t^{\prime}\right)\right\|_{L^{\infty}}^{2} d t^{\prime}\right)
$$

with $C$ a constant depending only on $\Delta_{0}, \mathcal{R}_{0}$ and $t_{*}$.

To estimate the second derivatives of $\mathbf{R}$ we apply (57) to the tensor $U=\mathbf{D R}$. Thus,

$$
\begin{aligned}
\int_{\Sigma_{t}}\left|\mathbf{D}^{2} \mathbf{R}\right|^{2} & \lesssim \int_{t_{0}}^{t} \int_{\Sigma_{t^{\prime}}}\left(\left|\mathbf{D}^{2} \mathbf{R}\right||\square(\mathbf{D R})|+|\mathbf{R}||\mathbf{D} \mathbf{R}|\left|\mathbf{D}^{2} \mathbf{R}\right|+\Delta_{0}\left|\mathbf{D}^{2} \mathbf{R}\right|^{2}\right) \\
& \lesssim \int_{t_{0}}^{t} \int_{\Sigma_{t^{\prime}}}\left(|\mathbf{R}||\mathbf{D} \mathbf{R}|\left|\mathbf{D}^{2} \mathbf{R}\right|+\Delta_{0}\left|\mathbf{D}^{2} \mathbf{R}\right|^{2}\right)
\end{aligned}
$$

Hence,

$$
\begin{aligned}
\left\|\mathbf{D}^{2} \mathbf{R}(t)\right\|_{L^{2}}^{2} \lesssim & \left\|\mathbf{D}^{2} \mathbf{R}\left(t_{0}\right)\right\|_{L^{2}}^{2}+\Delta_{0} \int_{t_{0}}^{t}\left\|\mathbf{D}^{2} \mathbf{R}\left(t^{\prime}\right)\right\|_{L^{2}}^{2} d t^{\prime} \\
& +\int_{t_{0}}^{t}\left\|\mathbf{D}^{2} \mathbf{R}\left(t^{\prime}\right)\right\|_{L^{2}}\left\|\mathbf{D} \mathbf{R}\left(t^{\prime}\right)\right\|_{L^{2}}\left\|\mathbf{R}\left(t^{\prime}\right)\right\|_{L^{\infty}} d t^{\prime} \\
\lesssim & \left\|\mathbf{D}^{2} \mathbf{R}\left(t_{0}\right)\right\|_{L^{2}}^{2}+\Delta_{0} \int_{t_{0}}^{t}\left\|\mathbf{D}^{2} \mathbf{R}\left(t^{\prime}\right)\right\|_{L^{2}}^{2} d t^{\prime} \\
& +\int_{t_{0}}^{t}\left\|\mathbf{D}^{2} \mathbf{R}\left(t^{\prime}\right)\right\|_{L^{2}}\left\|\mathbf{D R}\left(t^{\prime}\right)\right\|_{L^{2}}\left\|\mathbf{R}\left(t^{\prime}\right)\right\|_{L^{\infty}} d t^{\prime} .
\end{aligned}
$$


We therefore deduce the following

Proposition 3.8. Under the same assumptions as in Proposition 3.8, we have

$$
\left\|\mathbf{D}^{2} \mathbf{R}(t)\right\|_{L^{2}}^{2} \leq C\left(\left\|\mathbf{D}^{2} \mathbf{R}\left(t_{0}\right)\right\|_{L^{2}}^{2}+\int_{t_{0}}^{t}\left\|\mathbf{D R}\left(t^{\prime}\right)\right\|_{L^{2}}^{2}\left\|\mathbf{R}\left(t^{\prime}\right)\right\|_{L^{\infty}}^{2} d t^{\prime}\right)
$$

with $C$ a constant depending only on $\Delta_{0}, \mathcal{R}_{0}$ and $t_{*}$.

\section{Past null Boundaries}

The goal of this section is to review the main result of [Kl-Ro4], concerning the null boundaries of past causal domains, and show how they apply to our situation. Starting with any point $p$ in a subset $\mathcal{M}_{*}=\bigcup_{t \in\left[t_{0}, t_{*}\right)} \Sigma_{t}$ of $\mathbf{M}$, we denote by $\mathcal{J}^{-}(p)=\mathcal{J}^{-}\left(p ; \mathcal{M}_{*}\right)$ the causal past of $p$, relative to $\mathcal{M}_{*}$, by $\mathcal{I}^{-}(p)$ its interior and by $\mathcal{N}^{-}(p)$ its null boundary. In general $\mathcal{N}^{-}(p)$ is an achronal, Lipschitz hypersurface, ruled by the set of past null geodesics from $p$. We parametrize these geodesics with respect to the future, unit, time-like vector $\mathbf{T}_{p}$. Then, for every direction $\omega \in \mathbb{S}^{2}$, with $\mathbb{S}^{2}$ denoting the standard sphere in $\mathbb{R}^{3}$, consider the null vector $\ell_{\omega}$ in $T_{p} \mathbf{M}$,

$$
\mathbf{g}\left(\ell_{\omega}, \mathbf{T}_{p}\right)=1
$$

and associate to it the past null geodesic $\gamma_{\omega}(s)$ with initial data $\gamma_{\omega}(0)=p$ and $\dot{\gamma}_{\omega}(0)=\ell_{\omega}$. We further define a null vector field $L$ on $\mathcal{N}^{-}(p)$ according to

$$
L\left(\gamma_{\omega}(s)\right)=\dot{\gamma}_{\omega}(s) .
$$

$L$ may only be smooth almost everywhere on $\mathcal{N}^{-}(p)$ and can be multi-valued on a set of exceptional points. We can choose the parameter $s$ in such a way so that $L=\dot{\gamma}_{\omega}(s)$ is geodesic and $L(s)=1$.

For a sufficiently small $\delta>0$ the exponential map $\mathcal{G}=\mathcal{G}_{p}^{-}$, defined by

$$
(s, \omega) \rightarrow \gamma_{\omega}(s),
$$

is a diffeomorphism from $(0, \delta) \times \mathbb{S}^{2}$ to its image in $\mathcal{N}^{-}(p)$. Moreover for each $\omega \in \mathbb{S}^{2}$, either $\gamma_{\omega}(s)$ can be continued for all positive values 11 of $s$ or there exists a value $s_{*}(\omega)$ beyond which the points $\gamma_{\omega}(s)$ are no longer on the boundary $\mathcal{N}^{-}(p)$ of $\mathcal{J}^{-}(p)$ but rather in its interior; see [HE]. We call such points terminal points of $\mathcal{N}^{-}(p)$. We say that a terminal point $q=\gamma_{\omega}\left(s_{*}\right)$ is a conjugate terminal point if the map $\mathcal{G}$ is singular at $\left(s_{*}, \omega\right)$. A terminal point $q=\gamma_{\omega}\left(s_{*}\right)$ is said to be a cut locus terminal point if the map $\mathcal{G}=\mathcal{G}_{p}^{-}$is nonsingular at $\left(s_{*}, \omega\right)$ and there exists another null geodesic from $p$, passing through $q$.

Thus $\mathcal{N}^{-}(p)$ is a smooth manifold at all points except the vertex $p$ and the terminal points of its past null geodesic generators. We denote by $\mathcal{T}^{-}(p)$ the set of all terminal points and by $\dot{\mathcal{N}}^{-}(p)=\mathcal{N}^{-}(p) \backslash \mathcal{T}^{-}(p)$ the smooth portion of $\mathcal{N}^{-}(p)$. The set $\mathcal{G}^{-1}\left(\mathcal{T}^{-}(p)\right)$ has measure zero relative to the standard measure $d s d a_{\mathbb{S}^{2}}$ of the cone $[0, \infty) \times \mathbb{S}^{2}$, as it is a subset of the set $\left\{\left(s_{*}(\omega), \omega\right) \omega \in \mathbb{S}^{2}\right\}$. We will denote by $d A_{\mathcal{N}^{-}(p)}$ the corresponding measure on $\mathcal{N}^{-}(p)$. Observe that the definition is not intrinsic; it depends in fact on the normalization condition (60).

\footnotetext{
${ }^{11}$ for which $\gamma_{\omega}(s)$ stays in $\mathcal{M}_{*}$.
} 
Definition 4.1. Given $p \in \mathcal{M}_{*}$ we define $i_{*}^{-}(p)$ to be the supremum over all the values $s>0$ for which the exponential map $\mathcal{G}_{p}^{-}:(s, \omega) \rightarrow \gamma_{\omega}(s)$ is a global diffeomorphism. We shall refer to $i_{*}^{-}(p)$ as the past null radius of injectivity at $p$ relative to the geodesic foliation defined by (60).

We also define $i_{*}^{-}(p, t)$ (the null radius of injectivity relative to the $t$-foliation) to be the supremum over all the values $t(p)-t, t<t(p)$, for which the exponential $\operatorname{map} \mathcal{G}=\mathcal{G}_{p, t}^{-}$,

$$
(t, \omega) \rightarrow \gamma_{\omega}(t)=\gamma_{\omega}(s(t)),
$$

is a global diffeomorphism.

Definition 4.2. We define $d^{-}(p, t)$ to be the distance, measured with respect to the time parameter $t$, from $p$ to the past boundary of $\mathcal{M}_{*} \subset \mathbf{M}$.

The following theorem is an immediate consequence of the Main Theorem II proved in Kl-Ro4.

Theorem 4.3. Assume that $\mathcal{M}_{*}$ is globally hyperbolic and satisfies the assumptions A1 and A2 as well as (8). There exists a positive number $i_{*}>0$, depending only on $\Delta_{0}, \mathcal{R}_{0}$, and $t_{*}<0$, such that, for all $p \in \mathcal{M}_{*}$,

$$
i_{*}^{-}(p, t)>\min \left(i_{*}, d^{-}(p, t)\right) .
$$

Proof. According to the Main Theorem II of [Kl-Ro4] and the remark following it, $i_{*}$ depends only on our main constants, $\Delta_{0}, \mathcal{R}_{0}$ and a constant $N_{0}$ which provides uniform bounds for the lapse $n$,

$$
N_{0}^{-1} \leq n \leq N_{0} .
$$

The finiteness of $N_{0}, N_{0}^{-1}$ follows from (38) and the assumption $t_{*}<0$.

Remark 4.4. The proof of the lower bound on the radius of congugacy $i_{*}^{-}(p, t)$ in Kl-Ro1, Kl-Ro4 requires a smallness condition on the flux of curvature $\mathcal{F}(p, j(p))$ through the part of the null boundary $\mathcal{N}^{-}(p)$ defined by

$$
\mathcal{N}^{-}(p) \cap \bigcup_{t \in[t(p)-j(p), t(p)]} \Sigma_{t}, \quad j(p)=\min \left(i_{*}, d^{-}(p, t)\right) .
$$

The curvature flux $\mathcal{F}(p, j(p))$ is defined in Definition 4.13 As follows from Proposition 4.14, its smallness is implied by the smallness of the truncated energy

$$
\left(\int_{\Sigma_{t(p)-j(p)} \cap \mathcal{J}^{-}(p)} \mathcal{Q}(\mathbf{T}, \mathbf{T}, \mathbf{T}, \mathbf{T})\right)^{\frac{1}{2}} \leq \mathcal{R}_{0} .
$$

The constant $\mathcal{R}_{0}$, determined by the size of the initial data, is a priori not small and so neither is the truncated energy. The latter cannot even be made small by the choice of a small value of $j(p)$ as this would imply that the Bel-Robinson energy does not concentrate. However, a simple rescaling argument given below will show that the lower bound on the radius of injectivity in fact depends on the smallness of the scale-invariant quantity

$$
j(p) \mathcal{F}^{2}(p, j(p))
$$

and thus can easily be made small with the choice of $j(p) \leq c \mathcal{R}_{0}^{-2}$ for some universal small constant $c$. The rescaling argument proceeds as follows. Assume that we have 
already shown, as in Kl-Ro1, Kl-Ro4, that the condition

$$
\mathcal{F}^{2}(p, j(p))<c
$$

implies that

$$
i_{*}^{-}(p, t)>j(p) .
$$

We consider a family of space-times $\left(\mathcal{M}_{* \lambda}, \mathbf{g}_{\lambda}\right)$ defined according to the formula

$$
\mathbf{g}_{\lambda}(\tau, y)=\mathbf{g}(t(p)+\lambda(\tau-t(p)), \lambda y)
$$

This transformation dilates the null boundaries by a factor of $\lambda^{-1}$ so that the null boundary $\mathcal{N}_{\lambda}^{-}(p) \cap \bigcup_{\tau \in[t(p)-j, t(p)]} \Sigma_{\tau}$ for the space-time $\left(\mathcal{M}_{* \lambda}, \mathbf{g}_{\lambda}\right)$ corresponds to the set $\mathcal{N}^{-}(p) \cap \bigcup_{t \in[t(p)-\lambda j, t(p)]} \Sigma_{t}$ for $\left(\mathcal{M}_{*}, \mathbf{g}\right)$. Furthermore, $\left(\mathcal{M}_{* \lambda}, \mathbf{g}_{\lambda}\right)$ is a solution of the Einstein-vacuum equations and its null curvature flux $\mathcal{F}_{\lambda}(p, \lambda j)$ is related to the curvature flux $\mathcal{F}(p, j)$ of the metric $\mathbf{g}$ according to

$$
\mathcal{F}_{\lambda}^{2}(p, j)=\lambda \mathcal{F}^{2}(p, \lambda j) .
$$

Therefore, choosing $\lambda$ sufficiently small so that $\lambda \mathcal{F}^{2}(p, \lambda j)<c$ we conclude that the radius of injectivity $i_{* \lambda}^{-}(p, t)$ of the cone $\mathcal{N}_{\lambda}^{-}(p)$ satisfies $i_{*}^{-}(p, t)>\min (j$, $\left.\lambda^{-1} d^{-}(p, t)\right)$. This implies that the radius of injectivity $i_{*}^{-}(p, t)$ of the cone $\mathcal{N}^{-}(p)$ of the original metric $\mathbf{g}$ satisfies $i_{*}{ }^{-}(p, t)>\min \left(\lambda j, d^{-}(p, t)\right)$. The desired statement follows with the smallness condition

$$
\lambda j \mathcal{F}^{2}(p, \lambda j)<c j .
$$

Here $j$ can be chosen to be a universal small constant.

Once we have a lower bound for $i_{*}^{-}(p, t)$ it is straightforward to also get a lower bound for the radius of injectivity $i_{*}^{-}(p)$ with respect to the geodesic foliation. Indeed all we need is to show that $s$ does not vary much (along $\mathcal{N}^{-}(p)$ ) as a function of $t$ in a time interval of size 1. This follows immediately from the following.

Lemma 4.5. There exists a constant $c>0$, depending only on $\Delta_{0}$, such that

$$
c^{-1} \leq\left|\frac{d t}{d s}\right| \leq c .
$$

Proof. We introduce the null lapse,

$$
\varphi^{-1}=g(\mathbf{T}, L) .
$$

Observe that $\varphi>0$ with $\varphi(p)=1$. Moreover

$$
\frac{d t}{d s}=-(n \varphi)^{-1}
$$

with $n$ the lapse function of the $t$-foliation. On the other hand, we have

$$
L=-\varphi^{-1}(\mathbf{T}+N)
$$

with $N$ of length 1 perpendicular to $\mathbf{T}$. Now,

$$
\begin{aligned}
\frac{d}{d s} \varphi^{-1} & =\frac{d}{d s} \mathbf{g}(\mathbf{T}, L)=\mathbf{g}\left(\mathbf{D}_{L} \mathbf{T}, L\right)=-\frac{1}{2}{ }^{(\mathbf{T})} \pi_{L L} \\
& =\frac{1}{2} \varphi^{-2}\left({ }^{(\mathbf{T})} \pi_{\mathbf{T} N}+\frac{1}{2}{ }^{(\mathbf{T})} \pi_{N N}\right) .
\end{aligned}
$$

Therefore,

$$
\left|\frac{d}{d s} \varphi^{-1}\right| \lesssim \varphi^{-2} \Delta_{0}
$$


from which

$$
|\varphi(s)-1| \lesssim \Delta_{0} s
$$

Thus, for an interval in $s$ of size 1 we deduce that $2^{-1} \leq \phi(s) \leq 2$ and therefore, in view of the uniform bound for $n$ of Proposition 2.1, we infer that there must exist a constant $c>0$, depending only on $\Delta_{0}$, such that (64) holds.

Remark 4.6. We note that in $\mathrm{Kl}-\mathrm{Ro} 4$ the proof of the lower bound on the radius of injectivity $i_{*}^{-}(p)$ relied on the additional assumption that $\left|\partial_{t} n\right| \leq \Delta_{0}$. Below we provide the argument indicating that this assumption is implied by the conditions of this paper. In the case of the CMC foliation the lapse $n$ satisfies the equation

$$
\Delta n=|k|^{2} n-1 \text {. }
$$

Commuting the equation with $\partial_{t}$ we obtain

$$
-\Delta\left(\partial_{t} n\right)+|k|^{2}\left(\partial_{t} n\right)=P \text {. }
$$

Using our assumptions $|k|+|\nabla n| \leq \Delta_{0}$, the evolution equations (32), (33) for $g_{i j}$ and $k_{i j}$ and the elliptic estimates of the Appendix, we can easily show that the error term $P$ has the property that

$$
\|P\|_{L^{2}\left(\Sigma_{t}\right)} \leq C
$$

with $C$ dependent on $t_{*}, \mathcal{R}_{0}$ and $\Delta_{0}$. In view of the elliptic estimates of the Appendix, we then deduce that

$$
\left\|\nabla^{2}\left(\partial_{t} n\right)\right\|_{L^{2}\left(\Sigma_{t}\right)}+\left\|\partial_{t} n\right\|_{L^{2}\left(\Sigma_{t}\right)} \leq C
$$

from which we obtain the bound $\left|\partial_{t} n\right| \leq C$ by Corollary 2.10 ,

4.7. Geometry of smooth null cones. In this subsection we provide additional geometric information for the null boundaries $\mathcal{N}^{-}(p, \delta)$ with $\delta<i_{*}^{-}(p, t)$ with $i_{*}^{-}(p, t)$ a lower bound for past null injectivity radius with respect to the $t$-foliation. Here $\mathcal{N}^{-}(p, \delta)$ denotes the portion of $\mathcal{N}^{-}(p)$ for $t$ between $t(p)$ and $t(p)-\delta$.

Let $S_{t}$ denote the 2-dimensional space-like surfaces of intersection between $\Sigma_{t}$ and $\mathcal{N}^{-}(p)$. At any point of $\mathcal{N}^{-}(p, \delta) \backslash\{p\}$ we can define a conjugate null vector $\underline{L}$ with $\mathrm{g}(L, \underline{L})=-2$ and such that $\underline{L}$ is orthogonal to the leafs $S_{t}$. In addition we can choose $\left(e_{a}\right)_{a=1,2}$ tangent to $S_{t}$ such that together with $L$ and $\underline{L}$ we obtain a null frame,

$$
\begin{aligned}
& g(L, \underline{L})=-2, \quad \mathbf{g}(L, L)=\mathbf{g}(\underline{L}, \underline{L})=0, \\
& \mathbf{g}\left(L, e_{a}\right)=\mathbf{g}\left(\underline{L}, e_{a}\right)=0, \quad \mathbf{g}\left(e_{a}, e_{b}\right)=\delta_{a b} .
\end{aligned}
$$

We denote by $\gamma$ the restriction of $\mathbf{g}$ to $S_{t}$, i.e. $\gamma(X, Y)=\mathbf{g}(X, Y)$. Endowed with this metric, $S_{t}$ is a 2-dimensional compact Riemannian manifold. We denote by $\not \nabla$ the restriction of $\mathbf{D}$ to $S_{t}$. Clearly, for all $X, Y \in T\left(S_{t}\right)$,

$$
\not_{X} Y=\mathbf{D}_{X} Y+\frac{1}{2} \mathbf{g}\left(\mathbf{D}_{X} Y, \underline{L}\right) L+\frac{1}{2} \mathbf{g}\left(\mathbf{D}_{X} Y, L\right) \underline{L} .
$$

We recall, see [Kl-Ro1], the definitions of the following basic geometric quantities:

Definition 4.8. The null second fundamental forms $\chi, \underline{\chi}$, torsion $\zeta$ and the Ricci coefficient $\underline{\eta}$ of the foliation $S_{t}$ are defined as follows:

$$
\begin{array}{ll}
\chi_{a b}=\mathbf{g}\left(D_{a} L, e_{b}\right), & \underline{\chi}_{a b}=\mathbf{g}\left(D_{a} \underline{L}, e_{b}\right), \\
\zeta_{a}=\frac{1}{2} \mathbf{g}\left(D_{a} L, \underline{L}\right), & \underline{\eta}_{a}=\frac{1}{2} \mathbf{g}\left(e_{a}, D_{L} \underline{L}\right) .
\end{array}
$$


In addition 12 we define $\operatorname{tr} \chi=\gamma^{a b} \chi_{a b}, \hat{\chi}_{a b}=\chi_{a b}-\frac{1}{2} \operatorname{tr} \chi \gamma_{a b}$ and

$$
\omega=-\frac{1}{4} \mathbf{g}\left(D_{\underline{L}} \underline{L}, L\right), \quad \mu=\underline{L}(\operatorname{tr} \chi)+\frac{1}{2} \operatorname{tr} \chi \operatorname{tr} \underline{\chi}+2 \omega \operatorname{tr} \chi .
$$

We note that

$$
\begin{aligned}
& \underline{\chi}_{a b}=-\varphi^{2} \chi_{a b}+2 \varphi k_{a b}, \\
& \underline{\eta}_{a}=-\zeta_{a}-(n \varphi)^{-1} e_{a}(n \varphi), \\
& \omega=\varphi n^{-1} N(n) .
\end{aligned}
$$

Our conventions imply that

$$
\not_{X} Y=\mathbf{D}_{X} Y-\frac{1}{2} \underline{\chi}(X, Y) L-\frac{1}{2} \chi(X, Y) \underline{L} .
$$

We extend the definition of $\not \nabla$ to any covariant $S$-tangent tensor $\pi$ by the usual formula,

$$
\not_{X} \pi\left(Y_{1}, \ldots, Y_{k}\right)=X \pi\left(Y_{1}, \ldots, Y_{k}\right)-\pi\left(\nabla_{X} Y_{1}, \ldots, Y_{k}\right)-\ldots-\pi\left(Y_{1}, \ldots, \not_{X} Y_{k}\right)
$$

with $X, Y_{1}, \ldots, Y_{k} S$-tangent. Given an $S$-tangent vector field $X$ we define $\not_{L} X$ to be the projection to $S_{t}$ of $\mathbf{D}_{L} X$,

$$
\not_{L} X=\mathbf{D}_{L} X+\frac{1}{2} \mathbf{g}\left(\mathbf{D}_{L} X, \underline{L}\right) L
$$

We extend the definition to any covariant $S$-tangent tensor $\pi$ by

$$
\not_{L} \pi\left(Y_{1}, \ldots, Y_{k}\right)=L \pi\left(Y_{1}, \ldots, Y_{k}\right)-\pi\left(\mathbb{\nabla}_{L} Y_{1}, \ldots, Y_{k}\right)-\ldots-\pi\left(Y_{1}, \ldots, \mathbb{D}_{L} Y_{k}\right)
$$

with $Y_{1}, \ldots, Y_{k} S$-tangent.

Given an $S$-tangent tensor $\pi$ we write $\bar{\nabla} \pi=\left(\not \nabla \pi, \nabla_{L} \pi\right)$ and

$$
|\bar{\nabla} \pi|^{2}=\left|\not_{L} \pi\right|^{2}+|\not \nabla \pi|^{2}
$$

4.9. Tangential covariant derivatives of space-time tensors. In this section we make sense of covariant derivatives of space-time tensors, not necessarily $S$ tangent along a fixed surface $S=S_{t} \subset \mathcal{N}^{-}(p, \delta)$.

We start by defining a covariant derivative for a space-time vector $A_{\mu}$ defined on $S$. Thus we view $A$ as a section of a vector bundle $T^{*} \mathbf{M}$ over $S$. We interpret the covariant derivative $\not \nabla$ of $A$ along $S$ as a 1 -form on $S$ with values in $T^{*} \mathbf{M}$. Thus, for every vector field $X \in T S$ and any vector field $Z$ in $T \mathbf{M}$,

$$
\not \nabla A(X ; Z)=\not_{X} A(Z)=X(A(Z))-A\left(\mathbf{D}_{X} Z\right)=\mathbf{D}_{X} A(Z) .
$$

We also write

$$
\left(\not_{X} A\right)_{\mu}=X^{a} \mathbf{D}_{a} A_{\mu}, \quad \forall X \in T S .
$$

We define $\not^{2} A$, the second covariant derivatives of $A$ along $S$, by the formula $\not^{2} A(X, Y ; Z)=\left(\not_{X} \not \nabla A\right)(Y ; Z)=X(\not \nabla A(Y ; Z))-\not \nabla A\left(\not_{X} Y ; Z\right)-\not \nabla A\left(Y ; \mathbf{D}_{X} Z\right)$ or, for simplicity,

$$
\not^{2} A_{\mu}(X, Y)=\left(\mathbb{Z}_{Y}\left(\mathbb{Z}_{X} A\right)\right)_{\mu}-\left(\not_{\nabla_{Y} X} A\right)_{\mu}
$$

These definitions can be easily extended to higher covariant derivatives along $S$ and to higher-order tensors $A$.

\footnotetext{
${ }^{12}$ It is important to note that our calculations below do not depend on $\omega$.
} 
Given $A$ an $S$-tangent 1 -form on $M$ with values in $T M$ we define $\nabla_{\mathbf{L}} A(X ; Y)=L(A(X ; Y))-A\left(\nabla_{L} X ; Y\right)-A\left(X ; \mathbf{D}_{L} Y\right), \quad \forall X \in T S, \quad Y \in T M$. This definition extends naturally to higher-order tensors $A$. Note that for a scalar function $A$ on $M$ we have

$$
\nabla_{\mathbf{L}} A=\mathbf{D}_{\mathbf{L}} A
$$

4.10. Commutation formula. In what follows we will need the following commutation lemma; see [Kl-Ro1.

Lemma 4.11. Let $A_{\mu}$ be a function on $M$ with values in $T M$ satisfying the equation

$$
\nabla_{\mathbf{L}} A=F
$$

for some $T M$-valued function $F$. Then,

$$
\mathbb{\nabla}_{\mathbf{L}}\left(\nabla_{a} A_{\mu}\right)+\chi_{a b} \nabla_{b} A_{\mu}=\not_{a} F_{\mu}+\left(\zeta_{a}+\underline{\eta}_{a}\right) F_{\mu}+\mathbf{R}_{\mu}^{\lambda}{ }_{\mathbf{L} a}^{\lambda} A_{\lambda} .
$$

\subsection{Curvature flux.}

Definition 4.13. The curvature flux along $13 \mathcal{N}^{-}(p)$ is defined as follows:

$\mathcal{F}(p)=\left(\int_{\mathcal{N}^{-}(p)} \mathbf{Q}[\mathbf{R}](\mathbf{T}, \mathbf{T}, \mathbf{T}, L)\right)^{\frac{1}{2}}=\left(\int_{t_{0}}^{t(p)} n d t \int_{S_{t}} \mathbf{Q}[\mathbf{R}](\mathbf{T}, \mathbf{T}, \mathbf{T}, L) d A_{t}\right)^{\frac{1}{2}}$

with $d A_{t}$ the area element of $S_{t}$. We also let

$\mathcal{F}(p, \delta)=\left(\int_{\mathcal{N}^{-}(p, \delta)} \mathbf{Q}[\mathbf{R}](\mathbf{T}, \mathbf{T}, \mathbf{T}, L)=\int_{t(p)-\delta}^{t(p)} n d t \int_{S_{t}} \mathbf{Q}[\mathbf{R}](\mathbf{T}, \mathbf{T}, \mathbf{T}, L) d A_{t}\right)^{\frac{1}{2}}$

to be the curvature flux along $\mathcal{N}^{-}(p, \delta)$ for $\delta<i_{*}^{-}(p, t)$.

The following is an immediate consequence of the energy estimates of section 3.1. see also [Kl-Ro4.

Proposition 4.14. Under assumptions A2 as well as (8) the flux of curvature $\mathcal{N}^{-}(p)$ (denoted $\mathcal{F}(p)$ ) can be bounded by a uniform constant independent of $p$. More precisely, for all $p$ with $t_{0}<t(p) \leq t_{*}<0$,

$$
\mathcal{F}(p) \leq C\left(t_{*}, \Delta_{0}\right) \mathcal{R}_{0}
$$

where $C$ is the constant of Proposition 3.3 .

Proof. See section 5 in [Kl-Ro4].

We can also introduce the reduced flux, or geodesic curvature flux,

$$
\mathcal{R}(p)=\left(\int_{t_{0}}^{t(p)} \int_{S_{t}}|\alpha|^{2}+|\beta|^{2}+|\rho|^{2}+|\sigma|^{2}+|\underline{\beta}|^{2}\right)^{1 / 2}
$$

as well as

$$
\mathcal{R}(p, \delta)=\left(\int_{t(p)-\delta}^{t(p)} \int_{S_{t}}|\alpha|^{2}+|\beta|^{2}+|\rho|^{2}+|\sigma|^{2}+|\underline{\beta}|^{2}\right)^{1 / 2}
$$

\footnotetext{
${ }^{13}$ Given a scalar function $f$ on $\mathcal{N}^{-}(p)$ we denote its integral on $\mathcal{N}^{-}(p)$ to be $\int_{\mathcal{N}^{-}(p)} f=$ $\int_{t_{0}}^{t(p)} n d t \int_{S_{t}} f d A_{t}=\int_{\mathcal{N}^{-}(p)} f d A_{\mathcal{N}^{-}(p)}$.
} 
where $\alpha, \beta, \rho, \sigma, \beta, \underline{\alpha}$ are the null components of the Riemann curvature tensor relative to the $S_{t}$ foliation (note that the $\underline{\alpha}$ component of curvature does not appear in the expression for the curvature flux):

$$
\begin{aligned}
\alpha_{a b} & =\mathbf{R}_{L a L b}, \quad \beta_{a}=\frac{1}{2} \mathbf{R}_{a L \underline{L L}}, \quad \rho=\frac{1}{4} \mathbf{R}_{\underline{L L} \underline{L} L}, \\
\sigma & =\frac{1}{4}{ }^{\star} \mathbf{R}_{\underline{L L} \underline{L} L}, \quad \underline{\beta}_{a}=\frac{1}{2} R_{a \underline{L L} L}, \quad \underline{\alpha}_{a b}=\mathbf{R}_{\underline{L} a \underline{L} b} .
\end{aligned}
$$

Proposition 4.15. Under the same assumptions as in Proposition 4.14, we have, with a constant $C$ depending only on $t_{*}, \Delta_{0}$,

$$
\mathcal{R}(p) \leq C\left(t_{*}, \Delta_{0}\right) \mathcal{R}_{0} .
$$

Proof. We can express $L, \underline{L}$ in the form

$$
L=\varphi^{-1}(\mathbf{T}+N), \quad \underline{L}=\varphi(\mathbf{T}-N),
$$

where $N$ is the unit normal of $S_{t}$ on $\Sigma_{t}$ and $\varphi$ the null lapse defined by (65). Also,

$$
\mathbf{T}=\varphi L+\varphi^{-1} \underline{L} .
$$

Therefore,

$$
\begin{aligned}
\mathbf{Q}(\mathbf{T}, \mathbf{T}, \mathbf{T}, L)= & \varphi^{3} \mathbf{Q}(L, L, L, L)+3 \varphi^{2} \mathbf{Q}(\underline{L}, L, L, L) \\
& +3 \varphi \mathbf{Q}(\underline{L}, \underline{L}, L, L)+Q(\underline{L}, \underline{L}, \underline{L}, L) \\
= & \varphi^{3}|\alpha|^{2}+3 \varphi^{2}|\beta|^{2}+3 \varphi\left(\rho^{2}+|\sigma|^{2}\right)+|\underline{\beta}|^{2}
\end{aligned}
$$

and the result follows from the bound (67) for $\varphi$.

We can also get additional estimates for the flux associated to the first derivatives of the curvature tensor. To see that, we go back to the derivation of Theorem 3.7 We now integrate (55) in $\mathcal{J}^{-}(p)$ and derive

$$
\int_{\mathcal{N}^{-}(p)} \mathbf{Q}^{(w)}[\mathbf{R}](\mathbf{T}, L) \leq \int_{\Sigma_{t_{0}} \cap \mathcal{J}^{-}(p)} \mathbf{Q}^{(w)}[\mathbf{R}](\mathbf{T}, L)+\int_{\mathcal{J}^{-}(p)}\left|\mathbf{D}^{\beta}\left(\mathbf{Q}^{(w)}[\mathbf{R}]_{\alpha \beta} \mathbf{T}^{\alpha}\right)\right|
$$

Similarly,

$$
\begin{aligned}
\int_{\mathcal{N}^{-}(p, \delta)} \mathbf{Q}^{(w)}[\mathbf{R}](\mathbf{T}, L) \leq & \int_{\Sigma_{t(p)-\delta} \cap \mathcal{J}^{-}(p, \delta)} \mathbf{Q}^{(w)}[\mathbf{R}](\mathbf{T}, L) \\
& +\int_{\mathcal{J}^{-}(p, \delta)}\left|\mathbf{D}^{\beta}\left(\mathbf{Q}^{(w)}[\mathbf{R}]_{\alpha \beta} \mathbf{T}^{\alpha}\right)\right| .
\end{aligned}
$$

Here $\mathcal{J}^{-}(p)$ is the causal past of $p$ and $\mathcal{J}^{-}(p, \delta)$ the portion of $\mathcal{J}^{-}(p)$ to the future of $\Sigma_{t(p)-\delta}$. Now,

$$
\mathbf{Q}^{(w)}[\mathbf{R}](\mathbf{T}, L)=\mathbf{Q}^{(w)}[\mathbf{R}]\left(\varphi L+\varphi^{-1} \underline{L}, L\right)
$$

and

$$
\begin{aligned}
& \mathbf{Q}^{(w)}[\mathbf{R}](L, L)=\left|\mathbb{Z}_{L} \mathbf{R}\right|^{2} \\
& \mathbf{Q}^{(w)}[\mathbf{R}](\underline{L}, L)=|\not{R} \mathbf{R}|^{2} .
\end{aligned}
$$


We introduce the flux quantities,

$$
\begin{aligned}
\mathcal{F}^{(1)}(p) & =\left(\int_{\mathcal{N}^{-}(p)}\left(\left.|\not| \mathbf{R}\right|^{2}+\left|\mathbb{Z}_{L} \mathbf{R}\right|^{2}\right)\right)^{\frac{1}{2}}, \\
\mathcal{F}^{(1)}(p, \delta) & =\left(\int_{\mathcal{N}^{-}(p, \delta)}\left(\left.|\not| \nabla \mathbf{R}\right|^{2}+\left|\mathbb{\not}_{L} \mathbf{R}\right|^{2}\right)\right)^{\frac{1}{2}} .
\end{aligned}
$$

We can therefore reformulate Proposition 3.7 as follows:

Theorem 4.16. Assume that A1, A2 hold true. Then, for any $0<\delta \leq i_{*}$, with $i_{*}>0$ defined by Theorem 4.3 ,

$$
\|\mathbf{D R}(t)\|_{L^{2}}+\sup _{p \in \Sigma_{t}} \mathcal{F}^{(1)}(p, \delta) \leq C\left(\|\mathbf{D R}(t-\delta)\|_{L^{2}}+\left(\int_{t-\delta}^{t}\left\|\mathbf{R}\left(t^{\prime}\right)\right\|_{L^{\infty}} d t^{\prime}\right)^{1 / 2}\right)
$$

with $C$ a constant depending only on $\Delta_{0}, \mathcal{R}_{0}, t_{*}$.

4.17. Estimates for the Ricci coefficients. In this section we state without proof a proposition concerning the regularity properties of the Ricci coefficients $\operatorname{tr} \chi, \hat{\chi}, \zeta$ and $\eta$ as well as the mass aspect function $\mu$ associated to the $S_{t}$-foliation. A similar result was proved for the corresponding quantities associated with the geodesic foliation in [Kl-Ro1]; see also [Wang. The methods used for the geodesic foliations can be easily adapted to prove the result below.

Proposition 4.18. For any $t \in(t(p)-\delta, t(p))$ with $\delta<i_{*}^{-}(p, t)$, the Ricci coefficients tr $\chi, \hat{\chi}, \zeta, \underline{\eta}$ and $\mu$ satisfy the following estimates:

$$
\begin{aligned}
& \sup _{S_{t}}\left|\operatorname{tr} \chi-\frac{2}{s(t)}\right|+\left\|\sup _{t \in(t(p)-\delta, t(p))}(t(p)-t)|\not \nabla \operatorname{tr} \chi|\right\|_{L_{\omega}^{2}} \leq C, \\
& \sup _{\omega \in \mathbb{S}^{2}} \int_{t(p)-\delta}^{t(p)}\left(|\hat{\chi}|^{2}+|\zeta|^{2}+|\underline{\eta}|^{2}\right)(t, \omega) d t \leq C, \quad\|\mu\|_{L^{2}(\mathcal{N}-(p, \delta))} \leq C
\end{aligned}
$$

with a constant $C$ depending only on $\Delta_{0}, t_{*}$ and the curvature flux $\left.\mathcal{R}(p, \delta)\right)$. Here the points $\mathcal{N}^{-}(p, \delta)$ are parametrized by the coordinates $(t, \omega)$ with $\omega \in \mathbb{S}^{2}$. The volume forms $d A_{\mathcal{N}^{-}(p, \delta)}$ on $\mathcal{N}^{-}(p, \delta)$ and $d S_{t}$ on $S_{t}$ are respectively equivalent to the expressions $(t(p)-t)^{2} d t d \sigma_{\mathbb{S}^{2}}$ and $(t(p)-t)^{2} d \sigma_{\mathbb{S}^{2}}$ with $d \sigma_{\mathbb{S}^{2}}$ denoting the standard volume form on $\mathbb{S}^{2}$. The notation $L_{\omega}^{2}$ above refers to the $L^{2}$-norm with respect to the measure $d \sigma_{\mathbb{S}^{2}}$. Finally, the quantities $(t(p)-t)$ and $s(t)$ are equivalent.

\section{KirchHoff-Sobolev PARAmetrix}

Earlier in this paper, see Propositions 3.7, 3.8, we were able to derive $L^{2}$ estimates for derivatives of the curvature tensor which depend on the additional assumption on the boundedness of the $L^{\infty}$-norm of the curvature tensor. To estimate the latter we rely on a special version of the Kirchhoff-Sobolev parametrix introduced in [Kl-Ro5]. 
5.1. Optical function. To make sense of our Kirchhoff-Sobolev formula we need to define an optical function 14 , in a neighborhood of $\mathcal{N}^{-}(p, \delta), 0<\delta<i_{*}^{-}(p, t)$, such that it vanishes identically on $\mathcal{N}^{-}(p, \delta)$. Here $p$ is an arbitrary point of $\mathcal{M}_{*}=$ $\bigcup_{t \in\left[t_{0}, t_{*}\right)} \Sigma_{t}$. We recall that we have assumed that $\mathcal{M}_{*}$ is globally hyperbolic with Cauchy hypersurface $\Sigma_{t_{0}}$. We define $u$ uniquely relative to the time-like vector $\mathbf{T}_{p}$ as follows:

Let $\epsilon>0$ be a small number and $\Gamma_{\epsilon}:(1-\epsilon, 1+\epsilon) \rightarrow \mathcal{M}_{*}$ denote the timelike geodesic from $p$ such that $\Gamma_{\epsilon}(1)=p$ and $\Gamma_{\epsilon}^{\prime}(1)=\mathbf{T}_{p}$. From every point $q$ of $\Gamma_{\epsilon}$ let $\mathcal{N}^{-}(q)$ be the boundary of the past set of $q$ and let $\mathcal{N}^{-}(q, \delta)$ be defined as before.

We now define $u$ to be the function, constant on each $\mathcal{N}^{-}(q, \delta)$, such that for $q=\Gamma(t)$,

$$
\left.u\right|_{\dot{\mathcal{N}}^{-}(q)}=t-1
$$

This defines a smooth function $u$ which vanishes on $\mathcal{N}^{-}(p, \delta)$ and satisfies the eikonal equation,

$$
\mathbf{g}^{\alpha \beta} \partial_{\alpha} u \partial_{\beta} u=0 .
$$

Observe that the vector field $L=\mathbf{g}^{\alpha \beta} \partial_{\beta} u \partial_{\alpha}$ is null, geodesic and satisfies the normalization condition,

$$
\mathbf{g}\left(L, \mathbf{T}_{p}\right)=\mathbf{T}_{p}(u)=1 .
$$

Thus $L$ is the same as the vector field $L$ defined earlier in section 4 ,

5.2. Main representation formula. We shall next state a result which was proved in [Kl-Ro5], concerning tensorial wave equations of the form $\square \Psi=F$, with $\Psi$ a $k$-covariant tensor field. Let $p \in \mathcal{M}_{*}$ and $\delta<i_{*}^{-}(p, t)$. Let $\mathbf{A}$ be a tensor field of the same order satisfying

$$
\mathbf{D}_{L} \mathbf{A}+\frac{1}{2} \mathbf{A} \operatorname{tr} \chi=0, \quad s \mathbf{A}(p)=\mathbf{J}_{0} \quad \text { on } \quad \mathcal{N}^{-}(p, \delta),
$$

where $\mathbf{J}_{0}$ is a fixed $k$-tensor at $p,\left|\mathbf{J}_{0}(p)\right| \leq 1$.

Theorem 5.3. Let $p \in \mathcal{M}_{*}$ and $\delta<i_{*}^{-}(p, t)$. Let $\Psi$ be a $k$-covariant tensor field vanishing identically for $t \leq t(p)-\delta$. Then, given $\mathbf{A}$ a solution to the transport equations (84) we have 15

$$
\begin{aligned}
4 \pi \Psi(p) \cdot \mathbf{J}_{0}= & -\int_{\mathcal{N}^{-}(p, \delta)}\left(\mathbf{A} \square \Psi-\frac{1}{2} \mathbf{A} \cdot \mathbf{R}(\cdot, \cdot, \underline{L}, L) \cdot \Psi\right)+\frac{1}{2} \int_{\mathcal{N}^{-}(p, \delta)} \mu \mathbf{A} \cdot \Psi \\
& +\int_{\mathcal{N}^{-}(p, \delta)}\left(\triangle \mathbf{A}+\zeta_{a} \not_{a} \mathbf{A}\right) \cdot \Psi .
\end{aligned}
$$

We apply the theorem to the tensor field $\Psi=f(t) \mathbf{R}$, where $0 \leq f \leq 1$ is a smooth function supported in the interval $[t(p), t(p)-\delta]$ and identically equal to 1 in the interval $[t(p), t(p)-\delta / 2]$. Since $\mathbf{R}$ satisfies (53), we have

$$
\square(f \mathbf{R})=f \mathbf{R} \star \mathbf{R}+(\square f) \mathbf{R}+2 \mathbf{D}^{\alpha} f \mathbf{D}_{\alpha} \mathbf{R}
$$

\footnotetext{
14 i.e., a function which satisfies 83 below.

${ }^{15}$ Here 4 denotes the angular Laplace-Beltrami operator on the 2-surfaces $S_{t}$.
} 
with $\mathbf{R} \star \mathbf{R}$ defined in (52). In view of the theorem above, we have the formulas

$$
\begin{aligned}
4 \pi \mathbf{R}(p) \cdot \mathbf{J}_{0} & =I(p)+J(p)+K(p)+L(p)+\mathcal{E}, \\
I(p) & =\int_{\mathcal{N}^{-}(p, \delta)} \mathbf{A} \cdot f(\mathbf{R} \star \mathbf{R}), \\
J(p) & =-\frac{1}{2} \int_{\mathcal{N}^{-}(p, \delta)} \mathbf{A} \cdot \mathbf{R}(\cdot, \cdot, \underline{L}, L) \cdot f \mathbf{R}, \\
K(p) & =\int_{\mathcal{N}^{-}(p, \delta)}\left(\not \mathbf{A}+\zeta_{a} \not_{a} \mathbf{A}\right) \cdot f \mathbf{R}, \\
L(p) & =\frac{1}{2} \int_{\mathcal{N}^{-}(p, \delta)} \mu \mathbf{A} \cdot f \mathbf{R}, \\
\mathcal{E} & =\int_{\mathcal{N}^{-}(p, \delta)}\left(\square f(\mathbf{A} \cdot \mathbf{R})+2 \mathbf{D}^{\alpha} f\left(\mathbf{A} \cdot \mathbf{D}_{\alpha} \mathbf{R}\right)\right) .
\end{aligned}
$$

5.4. Estimates for $I(p)$. We consider the orthonormal frame $E_{0}=\mathbf{T}, E_{1}, E_{2}, E_{3}$ which is well defined everywhere in a neighborhood of the vertex $p$. Clearly the norm $|U|$, of an arbitrary tensorfield $U$, defined according to definition (27), coincides with the square root of the sum of squares of all the components of the tensor relative to this orthonormal frame. It is easy to see that

$$
|\mathbf{A} \cdot(\mathbf{R} \star \mathbf{R})| \leq|\mathbf{A}| \cdot|\mathbf{R} \star \mathbf{R}| .
$$

On the other hand, if $e_{4}=L, e_{3}=\underline{L}$ is the null pair in (68) and if we denote by $\underline{\alpha}(\mathbf{R} \star \mathbf{R}), \underline{\beta}(\mathbf{R} \star \mathbf{R}), \rho(\mathbf{R} \star \mathbf{R}), \sigma(\mathbf{R} \star \mathbf{R}), \beta(\mathbf{R} \star \mathbf{R}), \underline{\alpha}(\mathbf{R} \star \mathbf{R})$ the null decomposition of $\mathbf{R} \star \mathbf{R}$, as a Weyl field, relative to the null pair $e_{3}, e_{4}$, we can easily check that

$$
\begin{aligned}
|\mathbf{R} \star \mathbf{R}|^{2} \lesssim & |\underline{\alpha}(\mathbf{R} \star \mathbf{R})|^{2}+|\underline{\beta}(\mathbf{R} \star \mathbf{R})|^{2}+|\rho(\mathbf{R} \star \mathbf{R})|^{2} \\
& +|\sigma(\mathbf{R} \star \mathbf{R})|^{2}+|\beta(\mathbf{R} \star \mathbf{R})|^{2}+|\alpha(\mathbf{R} \star \mathbf{R})|^{2} .
\end{aligned}
$$

Indeed if we denote the Weyl field $\mathbf{R} \star \mathbf{R}$ by $W$ and introduce its electric and magnetic parts $E_{i j}=W_{i 0 j 0}, H_{i j}={ }^{\star} W_{i 0 j 0}$, we have

$$
|W|^{2}=|E|^{2}+|H|^{2} \text {. }
$$

Indeed, we have

$$
\begin{array}{ll}
W_{i j k 0}=-\epsilon_{i j}^{s} H_{s k}, & { }^{\star} W_{i j k 0}=\epsilon_{i j}^{s} E_{s k}, \\
W_{i j k l}=-\epsilon_{i j s} \in_{k l t} E^{s t}, & { }^{\star} W_{i j k l}=-\epsilon_{i j s} \in_{k l t} H^{s t},
\end{array}
$$

On the other hand, in terms of the null decomposition of $W$, relative to $e_{1}, e_{2}, e_{3}, e_{4}$,

$$
\begin{array}{ll}
E_{a b}=\frac{1}{4} \alpha_{a b}+\frac{1}{4} \underline{\alpha}_{a b}-\frac{1}{2} \rho \delta_{a b}, & H_{a b}=-\frac{1}{4}^{\star} \alpha_{a b}+\frac{1}{4}^{\star} \underline{\alpha}_{a b}-\frac{1}{2} \sigma \delta_{a b}, \\
E_{a N}=\frac{1}{2} \underline{\beta}_{a}+\frac{1}{2} \beta_{a}, & H_{a N}=\frac{1}{2}^{\star} \underline{\beta}_{a}-\frac{1}{2}^{\star} \beta_{a}, \\
E_{N N}=\rho, & H_{N N}=\sigma .
\end{array}
$$

Hence,

$$
|W|^{2}=|E|^{2}+|H|^{2} \lesssim|\alpha|^{2}+|\beta|^{2}+|\rho|^{2}+|\sigma|^{2}+|\underline{\beta}|^{2}+|\underline{\alpha}|^{2}
$$

which proves (88). We now estimate the right-hand side of (88). Clearly any null component of $\mathbf{R} \star \mathbf{R}$ can be expressed as a quadratic expression in the null components of $\mathbf{R}$. We observe that no null component of $\mathbf{R} \star \mathbf{R}$ can be quadratic in $\underline{\alpha}$. This can be easily proved by a signature consideration. Indeed we assign signature 2 to $\alpha(\mathbf{R})$, signature 1 to $\beta(\mathbf{R})$, signature 0 to $\rho(\mathbf{R})$ and $\sigma(\mathbf{R})$, signature -1 to $\underline{\beta}(\mathcal{R})$ and signature -2 to $\underline{\alpha}(\mathbf{R})$. Similarly we assign signature 2 to $\alpha(\mathbf{R} \star \mathbf{R})$, signature 1 to $\beta(\mathbf{R} \star \mathbf{R})$, signature 0 to $\rho(\mathbf{R} \star \mathbf{R})$ and $\sigma(\mathbf{R} \star \mathbf{R})$, signature -1 to 
$\underline{\beta}(\mathcal{R} \star \mathbf{R})$ and signature -2 to $\underline{\alpha}(\mathbf{R} \star \mathbf{R})$. It is easy to check that in the algebraic formula expressing the null components of $\mathbf{R} \star \mathbf{R}$ in terms of a quadratic form in the null components of $\mathbf{R}$, the total signature of each term must be the same as the signature of the corresponding null component of $\mathbf{R} \star \mathbf{R}$. Thus,

$$
\underline{\alpha}(\mathbf{R} \star \mathbf{R})=(\mathbf{R} \star \mathbf{R})_{a 3 b 3}=\operatorname{Qr}[\underline{\alpha},(\rho, \sigma)]+\operatorname{Qr}[\underline{\beta}, \underline{\beta}],
$$

where $\operatorname{Qr}[$,$] denotes a simple quadratic expression in the null components of \mathcal{R}$. Similarly,

$$
\begin{aligned}
\underline{\beta}(\mathbf{R} \star \mathbf{R}) & =\operatorname{Qr}[\underline{\alpha}, \beta]+\operatorname{Qr}[\underline{\beta},(\rho, \sigma)], \\
\rho(\mathbf{R}), \sigma(\mathbf{R}) & =\operatorname{Qr}[\underline{\alpha}, \alpha]+\operatorname{Qr}[\underline{\beta}, \beta]+\operatorname{Qr}[(\rho, \sigma),(\rho, \sigma)], \\
\beta(\mathbf{R} \star \mathbf{R}) & =\operatorname{Qr}[\underline{\beta}, \alpha]+\operatorname{Qr}[(\rho, \sigma), \beta], \\
\alpha(\mathbf{R} \star \mathbf{R}) & =\operatorname{Qr}[(\rho, \sigma), \alpha]+\operatorname{Qr}[\beta, \beta] .
\end{aligned}
$$

We now introduce the notation

$$
\left(|\mathbf{R}|^{\dagger}\right)^{2}=|\alpha|^{2}+|\beta|^{2}+|\rho|^{2}+|\sigma|^{2}+|\underline{\beta}|^{2}
$$

and deduce the following inequality:

$$
|\mathbf{R} \star \mathbf{R}| \lesssim|\mathbf{R}|^{\dagger} \cdot|\mathbf{R}| .
$$

We are now ready to estimate the term $I(p)$. Using the bounds for $\mathcal{R}(p)$ of Theorem 4.15 and $\mathcal{R}(p, \delta) \leq \mathcal{R}(p)$, we derive

$$
\begin{aligned}
|I(p)| & \lesssim \int_{\mathcal{N}^{-}(p, \delta)}|\mathbf{A}||\mathbf{R}|^{\dagger} \cdot|\mathbf{R}| \\
& \lesssim\left(\int_{\mathcal{N}^{-}(p, \delta)}|\mathbf{R}|^{\dagger}\right)^{1 / 2}\left(\int_{\mathcal{N}^{-}(p, \delta)}|\mathbf{A}|^{2}|\mathbf{R}|^{2}\right)^{1 / 2} \\
& \lesssim \mathcal{R}(p, \delta)\left(\int_{t(p)-\delta}^{t(p)}\|\mathbf{R}(t)\|_{L^{\infty}}^{2}\|\mathbf{A}(t)\|_{L^{2}\left(S_{t}\right)}^{2} d t\right)^{1 / 2} \\
& \lesssim \mathcal{R}(p, \delta)\left(\int_{t(p)-\delta}^{t(p)}\|\mathbf{A}(t)\|_{L^{2}\left(S_{t}\right)}^{2}\right)^{1 / 2} \sup _{t(p)-\delta<t<t(p)}\|\mathbf{R}(t)\|_{L^{\infty}\left(S_{t}\right)} .
\end{aligned}
$$

We therefore have

$$
|I(p)| \lesssim \mathcal{R}(p, \delta)\|\mathbf{A}\|_{L^{2}\left(\mathcal{N}^{-}(p, \delta)\right)}\|\mathbf{R}\|_{L^{\infty}\left(\mathcal{N}^{-}(p, \delta)\right)}
$$

5.5. Estimate for $J(p)$. In view of the fact that

$$
\int_{\mathcal{N}^{-}(p, \delta)}|\mathbf{R}(\cdot, \cdot, \underline{L}, L)|^{2} \lesssim \mathcal{R}(p, \delta)^{2},
$$

we deduce, proceeding exactly as for $I$,

$$
|J(p)| \lesssim \mathcal{R}(p, \delta)\|\mathbf{A}\|_{L^{2}\left(\mathcal{N}^{-}(p, \delta)\right)}\|\mathbf{R}\|_{L^{\infty}\left(\mathcal{N}^{-}(p, \delta)\right)} .
$$

5.6. Estimates for $L(p)$. We proceed as follows:

$$
\begin{aligned}
|L(p)| & \lesssim \int_{\mathcal{N}^{-}(p, \delta)}|\mu||\mathbf{A}||\mathbf{R}| \lesssim\left(\int_{\mathcal{N}^{-}(p, \delta)}|\mu|\right)^{1 / 2}\left(\int_{\mathcal{N}^{-}(p, \delta)}|\mathbf{A}|^{2}|\mathbf{R}|^{2}\right)^{1 / 2} \\
& \leq\|\mu\|_{L^{2}\left(\mathcal{N}^{-}(p, \delta)\right)}\|\mathbf{A}\|_{L^{2}\left(\mathcal{N}^{-}(p, \delta)\right)}\|\mathbf{R}\|_{L^{\infty}\left(\mathcal{N}^{-}(p, \delta)\right)}
\end{aligned}
$$


5.7. The term $K(p)$. Integrating by parts (recall that $f$ is a function of $t$ alone) we rewrite $K(p)$ as follows:

$$
K(p)=-\int_{\mathcal{N}^{-}(p, \delta)} f \not \nabla \mathbf{A} \cdot \not \nabla \mathbf{R}+\int_{\mathcal{N}^{-}(p, \delta)} f \zeta_{a} \mathbf{D}_{a} \mathbf{A} \cdot \mathbf{R} .
$$

We now estimate as follows:

$$
\begin{aligned}
|K(p)| & \lesssim \int_{\mathcal{N}^{-}(p, \delta)}(|\not \nabla \mathbf{A}\|\not \mathbf{R}|+| \zeta\| \not \mathbf{A}||\mathbf{R}|) \\
& \lesssim\|\not \nabla A\|_{L^{2}\left(\mathcal{N}^{-}(p, \delta)\right)}\left(\|\not \nabla \mathbf{R}\|_{L^{2}\left(\mathcal{N}^{-}(p, \delta)\right)}+\|\zeta \mathbf{R}\|_{L^{2}\left(\mathcal{N}^{-}(p, \delta)\right)}\right) .
\end{aligned}
$$

Therefore,

$$
|K(p)| \lesssim\|\not \mathbf{A}\|_{L^{2}\left(\mathcal{N}^{-}(p, \delta)\right)}\left(\|\not \nabla \mathbf{R}\|_{L^{2}\left(\mathcal{N}^{-}(p, \delta)\right)}+\|\mathbf{R}\|_{L^{\infty}\left(\mathcal{N}^{-}(p, \delta)\right)}\|\zeta\|_{L^{2}\left(\mathcal{N}^{-}(p, \delta)\right)}\right) .
$$

Going back to (86) and using the estimates for $I, J, K, L$ obtained above, we derive

Proposition 5.8. The following estimate holds for all $p \in \mathcal{M}_{*}$ and $0<\delta<$ $i_{*}^{-}(p, t)$ :

$$
\begin{aligned}
|\mathbf{R}(p)| \lesssim & \mathcal{E}+\|\mathbf{R}\|_{L^{\infty}\left(\mathcal{N}^{-}(p, \delta)\right)}\|\mathbf{A}\|_{L^{2}\left(\mathcal{N}^{-}(p, \delta)\right)}\left(\mathcal{R}(p, \delta)+\|\mu\|_{L^{2}\left(\mathcal{N}^{-}(p, \delta)\right)}\right) \\
& +\|\mathbb{\nabla} \mathbf{A}\|_{L^{2}\left(\mathcal{N}^{-}(p, \delta)\right)}\left(\|\not \mathbf{R}\|_{L^{2}\left(\mathcal{N}^{-}(p, \delta)\right)}+\|\mathbf{R}\|_{L^{\infty}\left(\mathcal{N}^{-}(p, \delta)\right)}\|\zeta\|_{L^{2}\left(\mathcal{N}^{-}(p, \delta)\right)}\right)
\end{aligned}
$$

We now recall that, according to (77),

$$
\mathcal{R}(p, \delta) \lesssim \mathcal{R}(p) \leq C\left(t_{*}, \Delta_{0}\right) \mathcal{R}_{0}
$$

Furthermore, by (82),

$$
\|\mu\|_{L^{2}\left(\mathcal{N}^{-}(p, \delta)\right)} \leq C\left(t_{*}, \Delta_{0}, \mathcal{R}_{0}\right)
$$

and

$$
\begin{aligned}
\|\zeta\|_{L^{2}\left(\mathcal{N}^{-}(p, \delta)\right)} & =\left(\int_{t(p)-\delta}^{t(p)} n d t \int_{S_{t}}|\zeta|^{2} d A_{t}\right)^{\frac{1}{2}} \\
& \lesssim \delta \sup _{\omega_{\mathbb{S}}^{2}}\left(\int_{t(p)-\delta}^{t(p)}|\zeta(t, \omega)|^{2} d t\right)^{\frac{1}{2}} \\
& \leq \delta C\left(t_{*}, \Delta_{0}, \mathcal{R}_{0}\right) .
\end{aligned}
$$

Finally, in the next section, we will establish that

$$
\|(t(p)-t) \mathbf{A}\|_{L^{\infty}\left(\mathcal{N}^{-}(p, \delta)\right)} \leq C, \quad\|\not \nabla \mathbf{A}\|_{L^{2}\left(\mathcal{N}^{-}(p, \delta)\right)} \leq C
$$

with a constant $C=C\left(t_{*}, \Delta_{0}, \mathcal{R}_{0}\right)$. This in particular implies that

$$
\|\mathbf{A}\|_{L^{2}\left(\mathcal{N}^{-}(p, \delta)\right)} \leq \delta^{\frac{1}{2}} C\left(t_{*}, \Delta_{0}, \mathcal{R}_{0}\right) .
$$

Putting this all together we deduce

$$
\|\mathbf{R}(t)\|_{L^{\infty}} \lesssim \mathcal{E}+\delta^{\frac{1}{2}} \sup _{p \in \Sigma_{t}}\|\mathbf{R}\|_{L^{\infty}\left(\mathcal{N}^{-}(p, \delta)\right)}+\sup _{p \in \Sigma_{t}}\|\not \mathbf{R}\|_{L^{2}\left(\mathcal{N}^{-}(p, \delta)\right)} .
$$

On the other hand, according to (80), we have

$$
\sup _{p \in \Sigma_{t}}\|\not \nabla \mathbf{R}\|_{L^{2}\left(\mathcal{N}^{-}(p, \delta)\right)} \leq \sup _{p \in \Sigma_{t}} \mathcal{F}^{(1)}(p, \delta) \lesssim\|\mathbf{D R}(t-\delta)\|_{L^{2}}+\left(\int_{t-\delta}^{t}\left\|\mathbf{R}\left(t^{\prime}\right)\right\|_{L^{\infty}}^{2} d t^{\prime}\right)^{1 / 2} .
$$


Therefore,

$$
\|\mathbf{R}(t)\|_{L^{\infty}} \lesssim \mathcal{E}+\|\mathbf{D R}(t-\delta)\|_{L^{2}}+\delta^{\frac{1}{2}} \sup _{t^{\prime} \in(t-\delta, t)}\left\|\mathbf{R}\left(t^{\prime}\right)\right\|_{L^{\infty}} .
$$

5.9. Estimates for the error term $\mathcal{E}$. We first observe that

$$
|\square t| \lesssim 1, \quad|\mathbf{D} t| \lesssim 1
$$

Therefore, since $f^{\prime}$ and $f^{\prime \prime}$ vanish for $|t-t(p)| \leq \delta / 2$ and

$$
\left\|f^{\prime}\right\|_{L^{\infty}} \leq \delta^{-1}, \quad\left\|f^{\prime \prime}\right\|_{L^{\infty}} \leq \delta^{-2}
$$

we derive

$$
\begin{aligned}
& |\mathcal{E}| \lesssim \delta^{\frac{1}{2}}\|\mathbf{A}\|_{L^{2}\left(\mathcal{N}^{-}(p, \delta)\right)} \sup _{t^{\prime} \in[t-\delta, t-\delta / 2]}\left(\delta^{-2}\left\|\mathbf{R}\left(t^{\prime}\right)\right\|_{L^{2}\left(S_{t}^{\prime}\right)}+\delta^{-1}\left\|\mathbf{D R}\left(t^{\prime}\right)\right\|_{L^{2}\left(S_{t}^{\prime}\right)}\right) \\
& \lesssim \sup _{t^{\prime} \in[t-\delta, t-\delta / 2]}\left(\delta^{-1}\left\|\mathbf{R}\left(t^{\prime}\right)\right\|_{L^{2}\left(S_{t}^{\prime}\right)}+\left\|\mathbf{D R}\left(t^{\prime}\right)\right\|_{L^{2}\left(S_{t}^{\prime}\right)}\right) \\
& \lesssim \sup _{t^{\prime} \in[t-\delta, t-\delta / 2]}\left(\delta^{-1}\left(\left\|\mathbf{R}\left(t^{\prime}\right)\right\|_{L^{2}\left(\Sigma_{t^{\prime}}\right)}+\left\|\mathbf{D R}\left(t^{\prime}\right)\right\|_{L^{2}\left(\Sigma_{t^{\prime}}\right)}\right)+\left\|\mathbf{D}^{2} \mathbf{R}\left(t^{\prime}\right)\right\|_{L^{2}\left(\Sigma_{t^{\prime}}\right)}\right) .
\end{aligned}
$$

The last step is a simple trace theorem which can be proven by an integration by parts argument.

5.10. Final estimate. Returning to (95), taking a supremum in $t$ over an interval of size $\delta$ and using the Sobolev inequality of Corollary 2.10,

$$
\left\|\mathbf{R}\left(t^{\prime}\right)\right\|_{L^{\infty}} \leq C\left(\left\|\mathbf{R}\left(t^{\prime}\right)\right\|_{L^{2}}+\left\|\mathbf{D R}\left(t^{\prime}\right)\right\|_{L^{2}}+\left\|\mathbf{D}^{2} \mathbf{R}\left(t^{\prime}\right)\right\|_{L^{2}}\right),
$$

we obtain

Proposition 5.11. There exists a positive $\delta>0$, sufficiently small but depending only on $\Delta_{0}, \mathcal{R}_{0}, t_{*}$, such that the following estimate holds true:

$$
\|\mathbf{R}(t)\|_{L^{\infty}} \leq C \delta^{-1} \sup _{t^{\prime} \in[t-2 \delta, t-\delta / 2]}\left(\left\|\mathbf{R}\left(t^{\prime}\right)\right\|_{L^{2}}+\left\|\mathbf{D R}\left(t^{\prime}\right)\right\|_{L^{2}}+\left\|\mathbf{D}^{2} \mathbf{R}\left(t^{\prime}\right)\right\|_{L^{2}}\right)
$$

with $C$ a constant depending only on $\Delta_{0}, t_{*}, \mathcal{R}_{0}$.

We now return to Propositions 3.7 and 3.8 , Combining them with the proposition above, we deduce

$$
\begin{aligned}
\|\mathbf{D R}(t)\|_{L^{2}}^{2} \leq & C\left(\|\mathbf{D R}(t-\delta / 2)\|_{L^{2}}^{2}+\int_{t-\delta / 2}^{t}\left\|\mathbf{R}\left(t^{\prime}\right)\right\|_{L^{\infty}}^{2} d t^{\prime}\right) \\
\leq & C \delta^{-1} \sup _{t^{\prime} \in[t-\delta, t-\delta / 2]}\left(\left\|\mathbf{R}\left(t^{\prime}\right)\right\|_{L^{2}}+\left\|\mathbf{D R}\left(t^{\prime}\right)\right\|_{L^{2}}^{2}+\left\|\mathbf{D}^{2} \mathbf{R}\left(t^{\prime}\right)\right\|_{L^{2}}^{2}\right), \\
\left\|\mathbf{D}^{2} \mathbf{R}(t)\right\|_{L^{2}}^{2} \leq & C\left(\left\|\mathbf{D}^{2} \mathbf{R}(t-\delta)\right\|_{L^{2}}^{2}+\int_{t-\delta / 2}^{t}\left\|\mathbf{D R}\left(t^{\prime}\right)\right\|_{L^{2}}^{2}\left\|\mathbf{R}\left(t^{\prime}\right)\right\|_{L^{\infty}}^{2} d t^{\prime}\right) \\
\leq & C\left\|\mathbf{D}^{2} \mathbf{R}(t-\delta)\right\|_{L^{2}}^{2} \\
& +C \delta^{-2} \sup _{t^{\prime} \in[t-\delta, t-\delta / 2]}\left(\left\|\mathbf{R}\left(t^{\prime}\right)\right\|_{L^{2}}+\left\|\mathbf{D R}\left(t^{\prime}\right)\right\|_{L^{2}}^{2}+\left\|\mathbf{D}^{2} \mathbf{R}\left(t^{\prime}\right)\right\|_{L^{2}}^{2}\right)^{2} .
\end{aligned}
$$

Consequently, for some $C$ depending only on $\Delta_{0}, \mathcal{R}_{0}$ and $t_{*}$,

$$
\|\mathbf{R}(t)\|_{H^{2}} \lesssim C \delta^{-1} \sup _{t^{\prime} \in[t-\delta, t-\delta / 2]}\left\|\mathbf{R}\left(t^{\prime}\right)\right\|_{H^{2}},
$$

where

$$
\|\mathbf{R}(t)\|_{H^{2}}=\|\mathbf{R}(t)\|_{L^{2}}+\|\mathbf{D R}(t)\|_{L^{2}}+\left\|\mathbf{D}^{2} \mathbf{R}(t)\right\|_{L^{2}} .
$$


Iterating the estimate as many times as needed, in steps of size $\delta / 2$, we derive

Theorem 5.12. Assume that $(\mathbf{M}, \mathbf{g})$ is a globally hyperbolic extension of $\Sigma_{0}$ satisfying the assumptions $\mathbf{A} \mathbf{1}$ and $\mathbf{A 2}$. Let $\mathcal{M}_{*}=\bigcup_{t \in\left[-t_{0}, t_{*}\right)} \Sigma_{t} \subset \mathbf{M}$ with $t_{0}=-1$. There exists a constant $C>0$ depending only on $\Delta_{0}, t_{*}$ and initial data $\left\|\mathbf{R}\left(t_{0}\right)\right\|_{H^{2}}$ such that

$$
\sup _{t \in\left[t_{0}, t_{*}\right)}\|\mathbf{R}(t)\|_{H^{2}} \leq C
$$

\section{Proof of Main Theorem 1.1}

Theorem 5.12 established above provides us with global uniform bounds for the curvature tensor $\mathbf{R}$ and $L^{2}$-bounds for its first two covariant derivatives. Using elliptic estimates we can also derive $L^{2}$-bounds for the first three derivatives of the second fundamental form; see Theorem 8.7. To finish the proof of the Main Theorem we only need to apply the following local existence result.

Proposition 6.1. Let $\left(\Sigma_{*}, g, k\right)$ be initial data for the Einstein-vacuum equations satisfying the constraint equations. We assume that $\Sigma_{*}$ is compact and has constant mean curvature $\tau=g^{i j} k_{i j}=$ const $<0$. Let $R$ denote the Ricci curvature tensor of $g$. Then there exists a smooth future Cauchy development of $(\Sigma, g, k)$ containing the region $\bigcup_{t \in[\tau, \tau+\rho]} \Sigma_{t}$, where each $\Sigma_{t}$ is a constant mean curvature hypersurface (with mean curvature equal to $t$ ) and $\Sigma_{\tau}=\Sigma_{*}$. The constant $\rho$ here depends only on the diameter and radius of injectivity of $\Sigma_{*}$, the strictly negative constant $\tau(\tau<0)$ and the following constant:

$$
\begin{aligned}
\mathcal{R}_{*}= & \|R\|_{L^{2}\left(\Sigma_{*}\right)}+\|\nabla R\|_{L^{2}\left(\Sigma_{*}\right)}+\left\|\nabla^{2} R\right\|_{L^{2}\left(\Sigma_{*}\right)} \\
& +\|k\|_{L^{4}\left(\Sigma_{*}\right)}+\|\nabla k\|_{L^{2}\left(\Sigma_{*}\right)}+\left\|\nabla^{2} k\right\|_{L^{2}\left(\Sigma_{*}\right)}+\left\|\nabla^{3} k\right\|_{L^{2}\left(\Sigma_{*}\right)} .
\end{aligned}
$$

Proof. The proof requires a slight modification of the local existence Theorem 10.2.1 in $\mathrm{C}-\mathrm{K}$.

Theorem 5.12 above, combined with the bounds on the second fundamental form stated in Theorem 8.7, proved below in the Appendix, implies that for each hypersurface $\Sigma_{t} \subset \mathcal{M}_{*}$ with $t_{0} \leq t<t_{*}$, the constant $\mathcal{R}_{*}$, defined in (99), is uniformly bounded. On the other hand, Theorem 2.9 together with $L^{\infty}$-bounds on curvature implied by Theorem 5.12 guarantees a uniform bound for both the diameter and radius of injectivity of $\Sigma_{t}$ for $t_{0} \leq t<t_{*}$. As a consequence, under assumptions A1, A2, as long as $t_{*}<0$ we can construct a smooth globally hyperbolic CMC development containing the region $\bigcup_{t \in\left[t_{0}, t_{*}\right]} \Sigma_{t}$.

\section{Estimates for A}

Proposition 7.1. Let $\mathbf{A}$ be the tensor defined in (84). Then for all $0<\delta<$ $i_{*}^{-}(p, t)$,

$$
\|(t(p)-t) \mathbf{A}\|_{L^{\infty}\left(\mathcal{N}^{-}(p, \delta)\right)} \leq C\left(t_{*}, \Delta_{0}, \mathcal{R}_{0}\right) .
$$

Proof. Recall, see Definition 3.4, the convention $b \lesssim 1$ for an inequality of the form $b \leq C\left(t_{*}, \Delta_{0}, \mathcal{R}_{0}\right)$. We claim that it suffices to prove the proposition for the case when $\mathbf{A}_{\mu}$ is a vector field. The general case can be derived by a simple induction argument. Recall that we have

$$
\mathbf{D}_{L} \mathbf{A}+\frac{1}{2} \operatorname{tr} \chi \mathbf{A}=0
$$


with $(s \mathbf{A})$ prescribed to be $\mathbf{J}_{0}$ at the vertex $p$. In view of the identity $\frac{d t}{d s}=-(n \varphi)^{-1}$ as well as estimate (67), it suffices to prove the inequality

$$
\|s \mathbf{A}\|_{L^{\infty}\left(\mathcal{N}^{-}(p, \delta)\right)} \lesssim 1
$$

Letting $\mathbf{B}=s \mathbf{A}$ we have

$$
\mathbf{D}_{L} \mathbf{B}=-\frac{1}{2}\left(\frac{2}{s}-\operatorname{tr} \chi\right) \mathbf{B},\left.\quad \mathbf{B}\right|_{s=0}=\mathbf{J}_{0}
$$

Recall that

$$
|\mathbf{B}|^{2}=B_{0}^{2}+|\underline{B}|^{2}=2\left|B_{0}\right|^{2}+\langle\mathbf{B}, \mathbf{B}\rangle,
$$

where $B_{0}=\langle\mathbf{B}, \mathbf{T}\rangle, \underline{B}$ is the projection of $\mathbf{B}$ on the foliation $\Sigma_{t}$ and $\langle\mathbf{B}, \mathbf{B}\rangle=$ $\mathbf{g}^{\mu \nu} \mathbf{B}_{\mu} \mathbf{B}_{\nu}$. We shall first estimate $\langle\mathbf{B}, \mathbf{B}\rangle$ by observing that

$$
\frac{d}{d s}\langle\mathbf{B}, \mathbf{B}\rangle=-\left(\frac{2}{s}-\operatorname{tr} \chi\right)\langle\mathbf{B}, \mathbf{B}\rangle,
$$

which in turn implies that

$$
\frac{d}{d t}\langle\mathbf{B}, \mathbf{B}\rangle=n \varphi\left(\frac{2}{s}-\operatorname{tr} \chi\right)\langle\mathbf{B}, \mathbf{B}\rangle
$$

Therefore, since $|\langle\mathbf{B}, \mathbf{B}\rangle(0)| \lesssim|\mathbf{J}(0)| \lesssim 1$,

$$
|\langle\mathbf{B}(t), \mathbf{B}(t)\rangle| \leq|\langle\mathbf{B}(0), \mathbf{B}(0)\rangle| \exp \left(\int_{t}^{t(p)} n \varphi\left|\operatorname{tr} \chi-\frac{2}{s^{\prime}}\right| d t^{\prime}\right) \lesssim 1,
$$

where the last inequality follows from (67) and (81). Therefore, for all $t(p)-\delta<$ $t \leq t(p)$,

$$
|\langle\mathbf{B}(t), \mathbf{B}(t)\rangle| \lesssim 1
$$

We shall next derive a transport equation for $B_{0}$ using the fact that $\mathbf{B}=-B_{0} \mathbf{T}+\underline{B}$,

$$
\begin{aligned}
\frac{d}{d s} B_{0} & =\left\langle\mathbf{D}_{\mathbf{L}} \mathbf{B}, \mathbf{T}\right\rangle+\left\langle\mathbf{B}, \mathbf{D}_{\mathbf{L}} \mathbf{T}\right\rangle \\
& =\frac{1}{2}\left(\frac{2}{s}-\operatorname{tr} \chi\right) B_{0}+\left\langle\underline{B}, \mathbf{D}_{\mathbf{L}} \mathbf{T}\right\rangle .
\end{aligned}
$$

Observe that

$$
\left\langle\underline{B}, \mathbf{D}_{\mathbf{L}} \mathbf{T}\right\rangle=-\frac{1}{2} \varphi^{-1}\left(\left\langle\underline{B}, \mathbf{D}_{\mathbf{T}} \mathbf{T}+\mathbf{D}_{N} \mathbf{T}\right\rangle\right) .
$$

Therefore, recalling our condition (28),

$$
\left|\left\langle\underline{B}, \mathbf{D}_{T} \mathbf{T}+\mathbf{D}_{N} \mathbf{T}\right\rangle\right| \lesssim \Delta_{0}|\underline{B}| .
$$

Therefore,

$$
\frac{d}{d s}\left|B_{0}\right| \lesssim\left|B_{0}\right|+\Delta_{0}|\underline{B}| .
$$

On the other hand, from (102), $\left.\left|-B_{0}^{2}+\right| \underline{B}\right|^{2}|=|\langle\underline{B}, \underline{B}\rangle \mid \lesssim 1$, from which

$$
|\underline{B}| \lesssim \sqrt{1+B_{0}^{2}} \lesssim\left|B_{0}\right|+1 \text {. }
$$

Therefore,

$$
\frac{d}{d t}\left|B_{0}\right| \lesssim\left|B_{0}\right|+\Delta_{0}\left(\left|B_{0}\right|+1\right)
$$

from which we deduce the estimate

$$
\left|B_{0}\right| \lesssim 1
$$


Thus, together with (102), we derive

$$
|\mathbf{B}| \leq\left|B_{0}\right|+|\underline{B}| \lesssim 1,
$$

as desired.

\subsection{Estimates for $\not \nabla A$.}

Proposition 7.3. Let $\mathbf{A}$ be the tensor defined in (84). Then,

$$
\begin{gathered}
\left\|\sup _{t(p)-\delta \leq t \leq t(p)}(t(p)-t)^{\frac{3}{2}}|\not \mathbf{A}(t)|\right\|_{L_{\omega}^{2}} \lesssim 1, \\
\|\not \nabla \mathbf{A}\|_{L^{2}\left(\mathcal{N}^{-}(p, \delta)\right)} \lesssim 1 .
\end{gathered}
$$

Proof. In what follows we recall that points on $\mathcal{N}^{-}(p, \delta)$ are parametrized by the coordinates $(t, \omega)$ with $\omega \in \mathbb{S}^{2}$. According to Proposition 4.18 the volume forms $d A_{\mathcal{N}^{-}(p, \delta)}$ on $\mathcal{N}^{-}(p, \delta)$ and $d S_{t}$ on $S_{t}$ are respectively equivalent to the expressions $(t(p)-t)^{2} d t d \sigma_{\mathbb{S}^{2}}$ and $(t(p)-t)^{2} d \sigma_{\mathbb{S}^{2}}$ with $d \sigma_{\mathbb{S}^{2}}$ denoting the standard volume form on $\mathbb{S}^{2}$. Similarly equivalent are the quantities $(t(p)-t)$ and $s(t)$. The notation $L_{\omega}^{2}$ above refers to the $L^{2}$-norm with respect to the measure $d \sigma_{\mathbb{S}^{2}}$.

We begin by applying the results of Lemma 4.11 to the equation $\mathbf{D}_{\mathbf{L}} \mathbf{A}+\frac{1}{2} \operatorname{tr} \chi \mathbf{A}=$ 0 and derive

$$
\not_{\mathbf{L}}\left(\not_{a} A_{\mu}\right)+\chi_{a b} \not_{b} A_{\mu}=-\frac{1}{2} \not_{a}\left(\operatorname{tr} \chi \mathbf{A}_{\mu}\right)-\frac{1}{2}\left(\zeta_{a}+\underline{\eta}_{a}\right) \operatorname{tr} \chi \mathbf{A}_{\mu}+\mathbf{R}_{\mu}^{\lambda}{ }_{L a} A_{\lambda} .
$$

Therefore,

$$
\begin{aligned}
\not_{\mathbf{L}}\left(\mathbb{\nabla}_{a} \mathbf{A}_{\mu}\right)+\operatorname{tr} \chi\left(\mathbb{\nabla}_{a} \mathbf{A}_{\mu}\right)= & -\hat{\chi}_{a b} \not_{b} \mathbf{A}_{\mu}-\frac{1}{2}\left(\mathbb{\nabla}_{a} \operatorname{tr} \chi\right) \mathbf{A}_{\mu} \\
& -\frac{1}{2}\left(\zeta_{a}+\underline{\eta}_{a}\right) \operatorname{tr} \chi \mathbf{A}_{\mu}+\mathbf{R}_{\mu L a}^{\lambda} A_{\lambda},
\end{aligned}
$$

which we rewrite in the form

$$
\begin{aligned}
\not_{\mathbf{L}} \mathbf{U}_{a \mu}+\operatorname{tr} \chi \mathbf{U}_{a \mu} & =-\hat{\chi}_{a b} \mathbf{U}_{b \mu}+\mathbf{F}_{a \mu},\left.\quad \mathbf{U}\right|_{s=0}=0 \\
\mathbf{F}_{a \mu} & =-\frac{1}{2}\left(\not_{a} \operatorname{tr} \chi\right) \mathbf{A}_{\mu}-\frac{1}{2}\left(\zeta_{a}+\underline{\eta}_{a}\right) \operatorname{tr} \chi \mathbf{A}_{\mu}+\mathbf{R}_{\mu \mathbf{L} a}^{\lambda} A_{\lambda}
\end{aligned}
$$

with $\mathbf{U}_{a \mu}=\not_{a} \mathbf{A}_{\mu}$. Observe that

$$
|\mathbf{U}|^{2}=2\left|\mathbf{U}_{a 0}\right|^{2}+\mathbf{U}_{a \mu} \mathbf{U}_{a}^{\mu},
$$

where, abusing the notation, $\left|\mathbf{U}_{a 0}\right|^{2}=\sum_{a=1,2}\left(\mathbf{U}_{a 0}\right)^{2}$.

Then,

$$
\not_{\mathbf{L}} \mathbf{U}_{a 0}+\operatorname{tr} \chi \mathbf{U}_{a 0}=-\hat{\chi}_{a b} \mathbf{U}_{b 0}+\mathbf{F}_{a 0}+\frac{1}{2} \phi^{-1}\left(n^{-1} \not \not_{j} n+k_{N j}\right) \mathbf{U}_{a j} .
$$

As a consequence,

$$
\begin{aligned}
\not_{\mathbf{L}}\left(s^{2} \mathbf{U}_{a 0}\right) & =s^{2}\left(\left(\frac{2}{s}-\operatorname{tr} \chi \delta_{a b}-\hat{\chi}_{a b}\right) \cdot \mathbf{U}_{b 0}+\mathbf{F}_{a 0}+\frac{1}{2} \phi^{-1}\left(n^{-1} \not \nabla_{j} n+k_{N j}\right) \mathbf{U}_{a j}\right) \\
& :=s^{2} \mathbf{G} .
\end{aligned}
$$

Using that $\frac{d s}{d t}=-n \varphi$ and

$$
\frac{d}{d s}\left(s^{4}\left(\mathbf{U}_{a 0}\right)^{2}\right)=s^{4} \mathbf{g}\left(\mathbf{G}, \mathbf{U}_{a 0}\right)
$$


together with boundedness of $n$ and $\varphi$, we estimate in the range $t(p)-\delta \leq t \leq t(p)$,

$$
\begin{aligned}
\left\|\sup _{t} s(t)^{3}\left(\mathbf{U}_{a 0}\right)^{2}\right\|_{L_{\omega}^{1}} & \lesssim\left\|\sup _{t} s(t)^{-1} \int_{t}^{t(p)} s(\tau)^{4} \mathbf{g}\left(\mathbf{G}, \mathbf{U}_{a 0}\right) d \tau\right\|_{L_{\omega}^{1}} \\
& \left.\lesssim\left\|\sup _{t} s(t)^{-1} \int_{t}^{t(p)} s(\tau)^{\frac{5}{2}}|\mathbf{G}| d \tau\right\|\right|_{L_{\omega}^{2}}\left\|\sup _{\tau} s(\tau)^{3}\left|\mathbf{U}_{a 0}\right|^{2}\right\|_{L_{\omega}^{1}}^{\frac{1}{2}} \\
& \lesssim \epsilon^{-1}\left\|\sup _{t} s(t)^{-1} \int_{t}^{t(p)} s(\tau)^{\frac{5}{2}}|\mathbf{G}| d \tau\right\|_{L_{\omega}^{2}}^{2}+\epsilon\left\|\sup _{\tau} s(\tau)^{3}\left|\mathbf{U}_{a 0}\right|^{2}\right\|_{L_{\omega}^{1}} .
\end{aligned}
$$

To control $\left\|\sup _{t} s(t)^{-1} \int_{t}^{t(p)} s(\tau)^{\frac{5}{2}}|\mathbf{G}| d \tau\right\|_{L_{\omega}^{2}}$, we first estimate the integral

$$
J:=\left\|\sup _{t} s(t)^{-1} \int_{t}^{t(p)} s(\tau)^{\frac{5}{2}}\left|\left(\frac{2}{s(\tau)}-\operatorname{tr} \chi \delta_{a b}-\hat{\chi}_{a b}\right) \cdot \mathbf{U}_{b 0}\right| d \tau\right\|_{L_{\omega}^{2}}
$$

as follows:

$$
\begin{aligned}
J & \lesssim\left\|\sup _{t} \int_{t}^{t(p)}\left|\left(\frac{2}{s(\tau)}-\operatorname{tr} \chi-\hat{\chi}\right)\right| d \tau\right\|_{L_{\omega}^{\infty}}\left\|\sup _{\tau} s(\tau)^{\frac{3}{2}}\left|\mathbf{U}_{a 0}\right|\right\|_{L_{\omega}^{2}} \\
& \lesssim\left\|\sup _{t} s(t)^{\frac{1}{2}} \int_{t}^{t(p)}\left|\left(\frac{2}{s(\tau)}-\operatorname{tr} \chi-\hat{\chi}\right)\right|^{2} d \tau\right\|_{L_{\omega}^{\infty}}^{\frac{1}{2}}\left\|\sup _{\tau} s(\tau)^{\frac{3}{2}}\left|\mathbf{U}_{a 0}\right|\right\|_{L_{\omega}^{2}} \\
& \lesssim \delta^{\frac{1}{2}}\left\|\sup _{\tau} s(\tau)^{\frac{3}{2}}\left|\mathbf{U}_{a 0}\right|\right\|_{L_{\omega}^{2}},
\end{aligned}
$$

where the last inequality follows from (81) and (82). On the other hand, in view of (100), (81), for $t(p)-\delta \leq t, \tau \leq t(p)$,

$$
\begin{gathered}
\left\|\operatorname { s u p } _ { t } s ( t ) ^ { - 1 } \int _ { t } ^ { t ( p ) } s ( \tau ) ^ { \frac { 5 } { 2 } } \left|\not \operatorname{tr} \chi\left\|\mathbf{A}\left|d \tau\left\|_{L_{\omega}^{2}} \lesssim \delta^{\frac{1}{2}}\right\| \sup _{\tau}(t(p)-\tau)\right| \not \operatorname{tr} \chi\right\|_{L_{\omega}^{2}} \lesssim \delta^{\frac{1}{2}},\right.\right. \\
\left\|\sup _{t} s(t)^{-1} \int_{t}^{t(p)} s(\tau)^{\frac{5}{2}}|\zeta+\underline{\eta}\|\operatorname{tr} \chi\| \mathbf{A}| d \tau\right\|_{L_{\omega}^{2}} \\
\lesssim\left\|\sup _{\tau}(t(p)-\tau)\left|\operatorname{tr} \chi \|_{L^{\infty}} \int_{t}^{t(p)}\right| \zeta+\left.\underline{\eta}\right|^{2} d \tau d \omega \lesssim 1 .\right.
\end{gathered}
$$

Moreover, using Proposition 4.15, we have 16

$$
\begin{aligned}
\left\|\operatorname { s u p } _ { t } s ( t ) ^ { - 1 } \int _ { t } ^ { ( p ) } s ( \tau ) ^ { \frac { 5 } { 2 } } \left|\mathbf{R}_{\mu}^{\lambda}{ }_{L a}\|\mathbf{A} \mid d \tau\|_{L_{\omega}^{2}}\right.\right. & \lesssim\left\|\sup _{t} s(t)^{-\frac{1}{2}} \int_{t}^{t(p)}(t(p)-\tau)\left|\mathbf{R}_{\mu L a}^{\lambda}\right| d \tau\right\|_{L_{\omega}^{2}} \\
& \lesssim\left\|\mathbf{R}_{\mu L a}^{\lambda}\right\|_{L^{2}\left(\mathcal{N}^{-}\left(p, \delta_{*}\right)\right)}^{\frac{1}{2}} \lesssim \mathcal{R}_{0}^{\frac{1}{2}} .
\end{aligned}
$$

Note that it is the presence of an $L$-component in the Riemann curvature tensor $\mathbf{R}_{\mu}^{\lambda}{ }_{L a}$ which allows us to express it as a linear combination of the tangential terms $\alpha, \beta, \rho, \sigma, \beta$ entering into the expression for the curvature flux.

Finally,

$$
\left\|\sup _{t} s(t)^{-1} \int_{t}^{t(p)} s(\tau)^{\frac{5}{2}}\left|\varphi^{-1}\left(n^{-1} \nabla_{j} n+k_{N j}\right) \mathbf{U}_{a j}\right| d \tau\right\|_{L_{\omega}^{2}} \lesssim \Delta_{0} \delta\left\|\sup _{\tau} s(\tau)^{\frac{3}{2}}\left|\mathbf{U}_{a} \cdot\right|\right\|_{L_{\omega}^{2}} .
$$

Therefore,

$\left\|\sup _{t} s(t)^{3}\left|\mathbf{U}_{a 0}\right|^{2}\right\|_{L_{\omega}^{1}} \lesssim \epsilon^{-1}\left(1+s(t)^{2}+\mathcal{R}_{0}\right)+\left(\epsilon+\epsilon^{-1} \Delta_{0}^{2} s(t)^{2}\right)\left\|\sup _{\tau} s(t)^{3}\left|\mathbf{U}_{a \cdot}\right|^{2}\right\|_{L_{\omega}^{1}}$.

\footnotetext{
${ }^{16}$ In what follows, $\left|\mathbf{R}_{\mu}^{\lambda}{ }_{L a}\right|$ denotes the norm of a two-tensor $\operatorname{Riem}\left(\cdot, L, e_{a}, \cdot\right)$.
} 
Combining this with the similar estimate on $\mathbf{U}_{a \mu} \mathbf{U}_{a}^{\mu}$ we obtain

$$
\left\|\sup _{t(p)-\delta \leq t \leq t(p)}(t(p)-t)^{3}\left|\mathbf{U}_{a 0}\right|^{2}\right\|_{L_{\omega}^{1}} \lesssim 1+\mathcal{R}_{0}
$$

which gives (104). The argument above also provides the inequality

$(t(p)-t)\|\mathbf{U}\|_{L_{\omega}^{2}} \lesssim(t(p)-t)^{\frac{1}{2}}+\frac{1}{t(p)-t} \int_{t}^{t(p)}\left((t(p)-\tau)\left\|\mathbf{R}_{\mu}^{\lambda}{ }_{\mathbf{L} a}\right\|_{L_{\omega}^{2}}+\|\zeta+\underline{\eta}\|_{L_{\omega}^{2}}\right) d \tau$.

Setting

$$
\mathcal{V}(\tau)=(t(p)-\tau)\left\|\mathbf{R}_{\mu}^{\lambda} \mathbf{L} a\right\|_{L_{\omega}^{2}}+\|\zeta+\underline{\eta}\|_{L_{\omega}^{2}}
$$

and using the $L^{2}$ maximal function estimate, we obtain

$$
\|\mathbf{U}\|_{L^{2}\left(\mathcal{N}-\left(p, \delta_{t}\right)\right)} \lesssim 1+\left(\int_{t(p)-\delta}^{t(p)}|V(\tau)|^{2} d \tau\right)^{\frac{1}{2}}
$$

from which we easily conclude that

$$
\|\mathbf{U}\|_{L^{2}\left(\mathcal{N}^{-}\left(p, \delta_{t}\right)\right)} \lesssim 1+\mathcal{R}_{0}^{\frac{1}{2}}
$$

and hence (105).

\section{Appendix}

Recall that the curvature tensor $\mathbf{R}$ can be decomposed into its electric and magnetic parts $E, H$ as follows:

$$
E(X, Y)=\langle\mathbf{R}(X, \mathbf{T}) \mathbf{T}, Y\rangle, \quad H(X, Y)=\left\langle{ }^{\star} \mathbf{R}(X, \mathbf{T}) \mathbf{T}, Y\right\rangle
$$

with ${ }^{\star} \mathbf{R}$ the Hodge dual of $\mathbf{R}$. One can easily check that $E$ and $H$ are tangent, traceless 2-tensors, to $\Sigma_{t}$ and that $|\mathbf{R}|^{2}=|E|^{2}+|H|^{2}$. We easily check the formulas relative to an orthonormal frame $e_{0}=T, e_{1}, e_{2}, e_{3}$,

$$
\begin{aligned}
& \mathbf{R}_{a b c 0}=-\epsilon_{a b s} H_{s c}, \quad{ }^{\star} \mathbf{R}_{a b c 0}=\epsilon_{a b s} E_{s c}, \\
& \mathbf{R}_{a b c d}=\epsilon_{a b s} \in c d t E_{s t}, \quad{ }^{\star} \mathbf{R}_{a b c d}=-\epsilon_{a b s} \in_{c d t} H_{s t} .
\end{aligned}
$$

We recall below some of the main formulas involving $k, E$ and $H$ :

$$
\begin{aligned}
E_{i j}-R_{i j} & =\operatorname{tr} k k_{i j}-k_{i}^{s} k_{s j}, \\
H_{i j} & =\operatorname{curl} k_{i j} .
\end{aligned}
$$

where, for any given symmetric two-tensor $l$ of $\Sigma_{t}$ one defines

$$
\operatorname{curl} l_{i j}=\epsilon_{i}^{a b} \nabla_{a} l_{b j}+\epsilon_{j}^{a b} \nabla_{a} l_{i b}
$$

We also recall the constraint equation for $k$,

$$
\nabla^{j} k_{i j}-\nabla_{i} \operatorname{tr} k=0
$$

In the particular case when tr $k$ is constant, equations (111) and (112) form an elliptic Hodge system on $\Sigma_{t}$,

$$
\operatorname{div} k=0, \quad \operatorname{curl} k=H .
$$


8.1. Elliptic $L^{2}$-estimates for Hodge systems. Here we recall the following lemma concerning rank-2 symmetric Hodge systems on a 3-dimensional compact Riemannian manifold $\Sigma$.

Lemma 8.2. The following elliptic $L^{2}$-estimates hold on a 3-dimensional Riemannian manifold $\Sigma$.

i. Let $V$ be a symmetric traceless 2-tensor on $\Sigma$ satisfying,

$$
\operatorname{div} V=\rho, \quad \operatorname{curl} V=\sigma .
$$

Then,

$$
\int_{\Sigma}\left(|\nabla V|^{2}+3 R_{m n} V^{i m} V_{i}^{n}-\frac{1}{2} R|V|^{2}\right)=\int_{\Sigma}\left(|\sigma|^{2}+\frac{1}{2}|\rho|^{2}\right),
$$

where $R_{i j}$ is the Ricci curvature of $\Sigma$ and $R$ its scalar curvature.

ii. For a scalar $\phi$, we have

$$
\int_{\Sigma}\left|\nabla^{2} \phi\right|^{2}+\int_{\Sigma} R^{i j} \nabla_{i} \phi \nabla_{j} \phi=\int_{\Sigma}|\Delta \phi|^{2} .
$$

Proof. See Proposition 4.4.1 in $\mathrm{C}-\mathrm{K}$.

8.3. A priori estimates for $k$. We now apply Lemma 8.2 to the Hodge system (113) for $k$ on a fixed hypersurface $\Sigma=\Sigma_{t}, t<0$,

$\int_{\Sigma}\left(|\nabla k|^{2}+3\left(k^{2}\right)^{m n}\left(E_{m n}+\left(k^{2}\right)_{m n}-\operatorname{tr} k k_{m n}\right)-\frac{1}{2}|k|^{2}\left(|k|^{2}-(\operatorname{tr} k)^{2}\right)\right)=\int_{\Sigma}|H|^{2}$,

where $k_{m n}^{2}=k_{m}^{m^{\prime}} k_{m^{\prime} n}$ and $|k|^{2}=\operatorname{tr}\left(k^{2}\right)$. Interpreting $k$ as $3 \times 3$ symmetric matrices we can write

$$
\begin{aligned}
3\left(k^{2}\right)^{m n}\left(k^{2}\right)_{m n} & -3\left(k^{2}\right)^{m n}(k)_{m n} \operatorname{tr} k-\frac{1}{2}|k|^{2}\left(|k|^{2}-(\operatorname{tr} k)^{2}\right) \\
& =3 \operatorname{tr}\left(k^{4}\right)-\frac{1}{2}\left(\operatorname{tr} k^{2}\right)^{2}-3 \operatorname{tr}\left(k^{3}\right) \operatorname{tr} k+\frac{1}{2} \operatorname{tr}\left(k^{2}\right)(\operatorname{tr} k)^{2} .
\end{aligned}
$$

Observe that we have the pointwise inequality 17 for an arbitrary symmetric matrix $k, \operatorname{tr}\left(k^{4}\right) \geq \frac{1}{3}|k|^{4}$. Therefore, in the case of a maximal foliation, satisfying the condition $\operatorname{tr} k=0$, we have

$$
\int_{\Sigma}|\nabla k|^{2}+\frac{1}{2}|k|^{4} \leq \int_{\Sigma}|H|^{2}+\int_{\Sigma}|E||k|^{2} \leq \int_{\Sigma}|H|^{2}+|E|^{2}+\int_{\Sigma} \frac{1}{4}|k|^{4} .
$$

Similarly, for the constant mean curvature foliation $\Sigma_{t}$ with negative mean curvature $\operatorname{tr} k=t<0$,

$$
\int_{\Sigma}|\nabla k|^{2}+\frac{1}{2}|k|^{4} \leq \int_{\Sigma}|H|^{2}+\int_{\Sigma}|E||k|^{2} \leq \int_{\Sigma}|H|^{2}+|E|^{2}+\int_{\Sigma} \frac{1}{4}|k|^{4} .
$$

This proves the following:

Proposition 8.4. On any leaf $\Sigma$ of a maximal or a constant negative mean curvature foliation $\Sigma_{t}$, the second fundamental form $k$ satisfies the estimate

$$
\int_{\Sigma}|\nabla k|^{2}+\frac{1}{4}|k|^{4} \leq \int_{\Sigma}|H|^{2}+|E|^{2}=\int_{\Sigma}|\mathbf{R}|^{2} .
$$

On the other hand, in view of the energy estimate of Proposition 3.3. we derive

\footnotetext{
${ }^{17}$ Indeed diagonalizing $k$ it suffices to prove the inequality for arbitrary real numbers $a, b, c$, $3\left(a^{2}+b^{2}+c^{2}\right)^{2} \geq a^{4}+b^{4}+c^{4}$.
} 
Corollary 8.5. The following estimates hold true with a constant $C$ depending only on $\Delta_{0}$ and $t_{*}$ :

$$
\|\nabla k(t)\|_{L^{2}}+\|k(t)\|_{L^{4}} \leq C \mathcal{R}_{0} .
$$

8.6. Higher derivative estimates for $k$. To derive second derivative estimates for $k$ we rewrite the curl equation in (113) in the form

$$
\nabla_{i} k_{j m}-\nabla_{j} k_{i m}=\epsilon_{i j^{s}} H_{s m} .
$$

Differentiating we obtain

$$
\nabla^{i} \nabla_{i} k_{j m}-\nabla^{i} \nabla_{j} k_{i m}=\epsilon_{i j^{s}} \nabla^{i} H_{s m}
$$

or, symbolically,

$$
\Delta k=R \star k+\nabla H,
$$

where $R \star k$ is a quadratic expression with respect to the Ricci curvature $R$ of $\Sigma_{t}$ and $k$. Thus, since the Ricci curvature $R$ can be expressed in the form

$$
R_{i j}-k_{i a} k_{j}^{a}+\operatorname{tr} k k_{i j}=E_{i j},
$$

we derive

$$
|\Delta k| \leq|k|^{3}+|E||k|+|\nabla H|
$$

Therefore,

$$
\int_{\Sigma_{t}}|\Delta k|^{2} \leq \int_{\Sigma_{t}}\left(|k|^{6}+|E|^{2}|k|^{2}+|\nabla H|^{2}\right) .
$$

It is easy to see by a standard integration by parts argument that

$$
\int_{\Sigma_{t}}\left|\nabla^{2} k(t)\right|^{2} \lesssim \int_{\Sigma_{t}}|\Delta k|^{2}+\int_{\Sigma_{t}}\left(|R|^{2}|k|^{2}+|R||\nabla k|^{2}\right) .
$$

Consequently,

$$
\int_{\Sigma_{t}}\left|\nabla^{2} k\right|^{2} \lesssim \int_{\Sigma_{t}}\left(|k|^{6}+|E|^{2}|k|^{2}+|\nabla H|^{2}\right)+\int_{\Sigma_{t}}\left(|R|^{2}|k|^{2}+|R||\nabla k|^{2}\right) .
$$

Therefore, since $\|k\|_{L^{\infty}} \leq \Delta_{0}$,

$$
\begin{aligned}
\left\|\nabla^{2} k(t)\right\|_{L^{2}} \leq & \Delta_{0}^{2}\left(\|k(t)\|_{L^{4}}^{4}+\|\mathbf{R}\|_{L^{2}}^{2}\right)+\|\nabla H(t)\|_{L^{2}}^{2} \\
& +\|R(t)\|_{L^{\infty}}\|\nabla k(t)\|_{L^{2}}^{2} .
\end{aligned}
$$

It is easy to see that $\|\nabla H(t)\|_{L^{2}}^{2} \lesssim\|\mathbf{D R}(t)\|_{L^{2}}$. Also,

$$
\|R(t)\|_{L^{\infty}} \leq\|\mathbf{R}\|_{L^{\infty}}+\|k\|_{L^{\infty}}^{2} \lesssim\|\mathbf{R}(t)\|_{H^{2}}+\Delta_{0}^{2} .
$$

Therefore, in view of Theorem 5.12 and the bounds for $\|\nabla k(t)\|_{L^{2}}$ and $\|k(t)\|_{L^{4}}$ of Corollary 8.5, we derive from (119)

$$
\left\|\nabla^{2} k(t)\right\|_{L^{2}} \leq C
$$

with $C$ a constant depending only on $\Delta_{0}, t_{*}$ and $\mathcal{R}_{0}$.

Differentiating once more the equation for $\Delta k$ and proceeding in the same fashion, we can also derive similar bounds for the third derivatives of $k$. This proves the following. 
Theorem 8.7. The second fundamental form $k$ of the $t$-foliation satisfies the following estimate, for all $t_{0} \leq t<\mathbf{t}_{*}$ :

$$
\left\|\nabla^{3} k(t)\right\|_{L^{2}}+\left\|\nabla^{2} k(t)\right\|_{L^{2}}+\|\nabla k(t)\|_{L^{2}}+\|k(t)\|_{L^{4}} \leq C
$$

with $C$ a constant depending only on $\Delta_{0}, t_{*}$ and $\mathcal{R}_{0}$.

\section{REFERENCES}

[And] M. Anderson, On long-time evolution in general relativity and geometrization of 3manifolds, Comm. Math. Phys. 222 (2001), 533-567. MR1888088(2003d:53113)

[BKM] J. T. Beale, T. Kato, A. Majda, Remarks on the breakdown of smooth solutions for the 3-D Euler equations, Comm. Math. Phys. 94 (1984), 61-66. MR763762 (85j:35154)

[Br] Y. Choquét-Bruhat, Théorème d'existence pour certains systèmes d'équations aux dérivées partielles non linéaires, Acta Math. 88 (1952), 141-225. MR0053338 $(14: 756 \mathrm{~g})$

[C-K] D. Christodoulou, S. Klainerman, The global nonlinear stability of the Minkowski space, Princeton Math. Series 41, 1993. MR1316662 (95k:83006)

[Fried] F.G. Friedlander, The Wave Equation on a Curved Space-time, Cambridge University Press, 1976. MR0460898 (57:889)

[HE] S. W. Hawking, G. F. R. Ellis, The Large Scale Structure of Space-time, Cambridge: Cambridge University Press, 1973. MR0424186 (54:12154)

[EM1] D. Eardley, V. Moncrief, The global existence of Yang-Mills-Higgs fields in 4dimensional Minkowski space. I. Local existence and smoothness properties, Comm. Math. Phys. 83 (1982), no. 2, 171-191. MR649158 (83e:35106a)

[EM2] D. Eardley, V. Moncrief, The global existence of Yang-Mills-Higgs fields in 4dimensional Minkowski space. II. Completion of proof, Comm. Math. Phys. 83 (1982), no. 2, 193-212. MR649159 (83e:35106b)

[HKM] T. J. R. Hughes, T. Kato and J. E. Marsden, Well-posed quasi-linear second-order hyperbolic systems with applications to nonlinear elastodynamics and general relativity, Arch. Rational Mech. Anal. 63 (1977), 273-394. MR0420024 (54:8041)

[Kl] S. Klainerman. PDE as a unified subject, Special Volume Geom. Funct. Anal. (2000), 279-315. MR 1826256 (2002e:35001)

[Kl-Ma] S. Klainerman, M. Machedon, Finite Energy Solutions for the Yang-Mills Equations in $\mathbb{R}^{3+1}$, Annals of Math. (2) 142 (1995), 39-119. MR.1338675 (96i:58167)

[Kl-Ro1] S. Klainerman, I. Rodnianski, Causal geometry of Einstein-vacuum spacetimes with finite curvature flux, Inventiones Math. 159 (2005), 437-529. MR2125732 (2006e:58042)

[Kl-Ro2] S. Klainerman, I. Rodnianski, A geometric approach to Littlewood-Paley theory, Geom. Funct. Anal. 16 (2006), 126-163. MR.2221254 (2007e:58046)

[Kl-Ro3] S. Klainerman, I. Rodnianski, Sharp trace theorems for null hypersurfaces on Einstein metrics with finite curvature flux, Geom. Funct. Anal. 16 (2006), 164-229. MR2221255 (2007e:58047)

[Kl-Ro4] S. Klainerman, I. Rodnianski, On the radius of injectivity of null hypersurfaces, J. Amer. Math. Soc. 21 (2008), 775-795. MR2393426 (2009g:35326)

[Kl-Ro5] S. Klainerman, I. Rodnianski, A Kirchhoff-Sobolev parametrix for the wave equation and applications, Journ. of Hyperbolic Equ., 4 (2007), 401-433. MR 2339803 (2008j:35108)

[M] V. Moncrief, An integral equation for spacetime curvature in General Relativity, Newton Institute preprint, NI05086-GMR. Surv. Differ. Geom., Vol. 10, Int. Press, Somerville, MA, 2006. MR2408224 (2009h:53166)

[Sob] S. Sobolev, Méthodes nouvelle à resoudre le problème de Cauchy pour les équations linéaires hyperboliques normales, Matematicheskii Sbornik, vol. 1 (43) 1936, 31 -79.

[Wang] Q. Wang, Causal geometry of Einstein vacuum space-times. Ph.D. thesis, Princeton University, 2006. 
Department of Mathematics, Princeton University, Princeton, New Jersey 08544

E-mail address: seri@math.princeton.edu

Department of Mathematics, Princeton University, Princeton, New Jersey 08544

E-mail address: irod@math.princeton.edu 